\title{
Tropicalization of classical moduli spaces
}

Qingchun Ren

\author{
Steven V Sam
}

November 5, 2013

\begin{abstract}
The image of the complement of a hyperplane arrangement under a monomial map can be tropicalized combinatorially using matroid theory. We apply this to classical moduli spaces that are associated with complex reflection arrangements. Starting from modular curves, we visit the Segre cubic, the Igusa quartic, and moduli of marked del Pezzo surfaces of degrees 2 and 3. Our primary example is the Burkhardt quartic, whose tropicalization is a 3-dimensional fan in 39-dimensional space. This effectuates a synthesis of concrete and abstract approaches to tropical moduli of genus 2 curves.
\end{abstract}

\section{Introduction}

Algebraic geometry is the study of solutions sets to polynomial equations. Solutions that depend on an infinitesimal parameter can be analyzed combinatorially using min-plus algebra. This insight led to the development of tropical algebraic geometry [39]. While all algebraic varieties and their tropicalizations may be explored at various level of granularity, varieties that serve as moduli spaces are usually studied at the highest level of abstraction. This paper does exactly the opposite: we investigate and tropicalize certain concrete moduli spaces, mostly from the 19th century repertoire [36], by means of their defining polynomials.

A first example, familiar to all algebraic geometers, is the moduli space $\mathcal{M}_{0, n}$ of $n$ distinct points on the projective line $\mathbb{P}^{1}$. We here regard $\mathcal{M}_{0, n}$ as a subvariety in a suitable torus. Its tropicalization $\operatorname{trop}\left(\mathcal{M}_{0, n}\right)$ is a simplicial fan of dimension $n-3$ whose points parametrize all metric trees with $n$ labeled leaves. The cones distinguish different combinatorial types of metric trees. The defining polynomials of this (tropical) variety are the $\left(\begin{array}{l}n \\ 4\end{array}\right)$ Plücker quadrics $p_{i j} p_{k \ell}-p_{i k} p_{j \ell}+p_{i \ell} p_{j k}$. These quadrics are the $4 \times 4$-subpfaffians of a skew-symmetric $n \times n$ matrix, and they form a tropical basis for $\mathcal{M}_{0, n}$. The tropical compactification defined by this fan is the moduli space $\overline{\mathcal{M}}_{0, n}$ of $n$-pointed stable rational curves. The picture for $n=5$ is delightful: the tropical surface $\operatorname{trop}\left(\mathcal{M}_{0,5}\right)$ is the cone over the Petersen graph, with vertices labeled by the 10 Plücker coordinates $p_{i j}$ as in Figure 1.

A related example is the universal family $\mathcal{A}(5)$ over the modular curve $X(5)$. The relevant combinatorics goes back to Felix Klein and his famous 1884 lectures on the icosahedron [38]. 


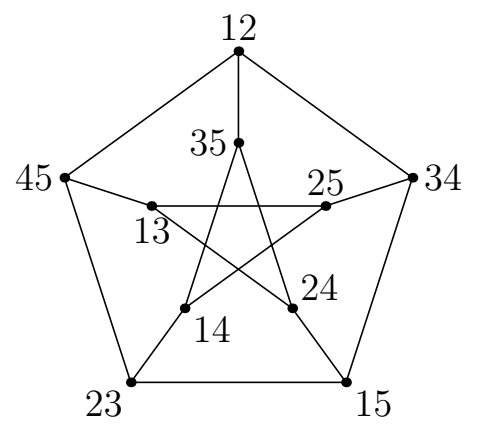

Figure 1: The Petersen graph represents the tropicalization of $\mathcal{M}_{0,5}$.

Following Fisher [25], the surface $\mathcal{A}(5)$ sits in $\mathbb{P}^{1} \times \mathbb{P}^{4}$ and has the Pfaffian representation

$$
\operatorname{rank}\left[\begin{array}{ccccc}
0 & -a_{1} x_{1} & -a_{2} x_{2} & a_{2} x_{3} & a_{1} x_{4} \\
a_{1} x_{1} & 0 & -a_{1} x_{3} & -a_{2} x_{4} & a_{2} x_{0} \\
a_{2} x_{2} & a_{1} x_{3} & 0 & -a_{1} x_{0} & -a_{2} x_{1} \\
-a_{2} x_{3} & a_{2} x_{4} & a_{1} x_{0} & 0 & -a_{1} x_{2} \\
-a_{1} x_{4} & -a_{2} x_{0} & a_{2} x_{1} & a_{1} x_{2} & 0
\end{array}\right] \leq 2 .
$$

The base of this family is $\mathbb{P}^{1}$ with coordinates $\left(a_{1}: a_{2}\right)$. The tropical surface $\operatorname{trop}(\mathcal{A}(5))$ is a fan in $\mathbb{P P}^{1} \times \mathbb{P P}^{4}$, which is combinatorially the Petersen graph in Figure 1. The central fiber, over the vertex of $\mathbb{T P}^{1}$ given by $\operatorname{val}\left(a_{1}\right)=\operatorname{val}\left(a_{2}\right)$, is the 1-dimensional fan with rays $e_{0}, e_{1}, e_{2}, e_{3}, e_{4}$. These correspond to the edges $34-25,12-35,45-13,23-14,15-24$. For $\operatorname{val}\left(a_{1}\right)<\operatorname{val}\left(a_{2}\right)$, the fiber is given by the pentagon 12-34-15-23-45-12 with these rays attached. For $\operatorname{val}\left(a_{1}\right)>\operatorname{val}\left(a_{2}\right)$, it is the pentagram 35-14-25-13-24-35 with the five rays. Each of the edges has multiplicity 5 . The map from $\operatorname{trop}(\mathcal{A}(5))$ onto $\mathbb{T P}^{1}$ is visualized in Figure 2.

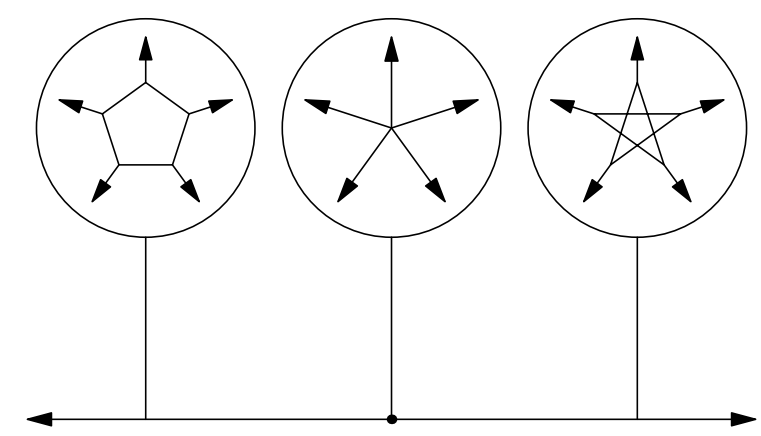

Figure 2: The universal family of tropical elliptic normal curves of degree 5.

The discriminant of our family $\mathcal{A}(5) \rightarrow \mathbb{P}^{1}$ is the binary form

$$
a_{1}^{11} a_{2}-11 a_{1}^{6} a_{2}^{6}-a_{1} a_{2}^{11},
$$

whose 12 zeros represent Klein's icosahedron. The modular curve $X(5)$ is $\mathbb{P}^{1}$ minus these 12 points. For each $\left(a_{1}: a_{2}\right) \in X(5)$, the condition (1.1) defines an elliptic normal curve in $\mathbb{P}^{4}$. 
Throughout this paper we work over an algebraically closed field $K$ of characteristic 0 . Our notation and conventions regarding tropical geometry follow [39]. For simplicity of exposition, we identify the tropical projective space $\mathbb{T P}^{n}$ with its open part $\mathbb{R}^{n+1} / \mathbb{R}(1,1, \ldots, 1)$.

The adjective "classical" in our title has two meanings. Classical as opposed to tropical refers to moduli spaces that are defined over fields, the usual setting of algebraic geometry. The foundations for tropicalizing such schemes and stacks are currently being developed, notably in the work of Abramovich et al. [3] and Baker et al. [7] (see [2] for a survey). These rest on the connection to nonarchimedean geometry. Classical as opposed to modern refers to moduli spaces that were known in the 19th century. We focus here on the varieties featured in Hunt's book [36], notably the Segre cubic, the Igusa quartic, the Burkhardt quartic, and their universal families. We shall also revisit the work on tropical del Pezzo surfaces by Hacking et al. in [33] and explain how this relates to the tropical Göpel variety of [44, §9].

Each of our moduli spaces admits a high-dimensional symmetric embedding of the form

$$
\mathbb{P}^{d} \stackrel{\text { linear }}{\hookrightarrow} \mathbb{P}^{m} \stackrel{\substack{\text { monomial } \\-\rightarrow}}{\mathbb{P}^{n}}
$$

The coordinates of the first map are the linear forms defining the $m+1$ hyperplanes in a complex reflection arrangement $\mathcal{H}$ in $\mathbb{P}^{d}$, while the coordinates of the second map are monomials that encode the symplectic geometry of a finite vector space. The relevant combinatorics rests on the representation theory developed in [29,30]. Each of our moduli spaces is written as the image of a map (1.3) whose coordinates are monomials in linear forms, and hence the formula in [20, Theorem 3.1] expresses its tropicalization using the matroid structure of $\mathcal{H}$.

Our warm-up example, the modular curve $X(5)$, fits the pattern (1.3) for $d=1, m=11$ and $n=5$. Its arrangement $\mathcal{H} \subset \mathbb{P}^{1}$ is the set of 12 zeros of (1.2), but now identified with the complex reflection arrangement $\mathrm{G}_{16}$ as in $[29, \S 2.2]$. If we factor (1.2) into six quadrics,

$$
\left(a_{1} a_{2}\right) \cdot \prod_{i=1}^{5}\left(\left(\gamma^{5-i} a_{1}+\left(\gamma+\gamma^{4}\right) a_{2}\right)\left(\gamma^{i} a_{1}+\left(\gamma^{2}+\gamma^{3}\right) a_{2}\right)\right.
$$

where $\gamma$ is a primitive fifth root of unity, then these define the coordinates of $\mathbb{P}_{-\rightarrow}^{11} \stackrel{\text { monomial }}{\rightarrow} \mathbb{P}^{5}$. The image is a quadric in a plane in $\mathbb{P}^{5}$, and $X(5)$ is now its intersection with the torus $\mathbb{G}_{m}^{5}$. The symmetry group $\mathrm{G}_{16}$ acts on $\mathbb{P}^{5}$ by permuting the six homogeneous coordinates. The tropical modular curve $\operatorname{trop}(X(5))$ is the standard one-dimensional fan in $\mathbb{T} \mathbb{P}^{5}$, with multiplicity five and pentagonal fibers as above. But now the full symmetry group acts on the surface $\mathcal{A}(5) \subset \mathbb{P}^{5} \times \mathbb{P}^{4}$ and the corresponding tropical surface by permuting coordinates.

We next discuss the organization of this paper. In Section 2 we study the Segre cubic and the Igusa quartic, in their symmetric embeddings into $\mathbb{P}^{14}$ and $\mathbb{P}^{9}$, respectively. We show that the corresponding tropical variety is the space of phylogenetic trees on six taxa, and we determine the universal family of tropical Kummer surfaces over that base. In Section 3 we study the Burkhardt quartic in its symmetric embedding in $\mathbb{P}^{39}$, and, over that base, we compute the universal family of abelian surfaces in $\mathbb{P}^{8}$ along with their associated tricanonical curves of genus 2. In Section 4 we compute the Bergman fan of the complex reflection arrangement $\mathrm{G}_{32}$ and from this we derive the tropical Burkhardt quartic in $\mathbb{T}^{39}$. The corresponding tropical compactification is shown to coincide with the Igusa desingularization of the Baily-Borel-Satake compactification of $\mathcal{A}_{2}(3)$. In Section 5 we relate our findings to 
the abstract tropical moduli spaces of $[11,15]$. Figure 3 depicts the resulting correspondence between trees on six taxa, metric graphs of genus 2, and cones in the tropical Burkhardt quartic. In Section 6 we study the reflection arrangements of types $E_{6}$ and $E_{7}$, and we show how they lead to the tropical moduli spaces of marked del Pezzo surfaces constructed by Hacking, Keel and Tevelev [33]. For $\mathrm{E}_{7}$ we recover the tropical Göpel variety of [44, §9]. This is a six-dimensional fan which serves as the universal family of tropical cubic surfaces.

\section{Acknowledgements}

Steven Sam was supported by a Miller Research Fellowship at UC Berkeley. Qingchun Ren and Bernd Sturmfels were supported by the National Science Foundation (DMS-0968882) and DARPA (HR0011-12-1-0011). We thank Florian Block, Dustin Cartwright, Melody Chan, Diane Maclagan, Sam Payne and Jenia Tevelev for helpful discussions. We are especially grateful to Gus Schrader for his contributions to the material in Section 3.

\section{Segre Cubic, Igusa Quartic, and Kummer Surfaces}

The moduli spaces in this section are based on the hyperplane arrangement in $\mathbb{P}^{4}$ associated with the reflection representation of the symmetric group $\Sigma_{6}$. It consists of the 15 hyperplanes

$$
x_{i}-x_{j}=0 \quad(1 \leq i<j \leq 6) \text {. }
$$

Here $\mathbb{P}^{4}$ is the projectivization of the 5 -dimensional vector space $K^{6} / K(1,1,1,1,1,1)$. The 15 linear forms in (2.1) define the map $\mathbb{P}^{4} \stackrel{\text { linear }}{\hookrightarrow} \mathbb{P}^{14}$ whose image is the 4-dimensional subspace $\mathrm{Cyc}_{4}$ of $\mathbb{P}^{14}$ that is defined by the linear equations $z_{i j}-z_{i k}+z_{j k}=0$ for $1 \leq i<j<k \leq 6$.

The corresponding tropical linear space $\operatorname{trop}\left(\mathrm{Cyc}_{4}\right)$, with the coarsest fan structure, is isomorphic to both the moduli space of equidistant (rooted) phylogenetic trees with 6 vertices and the moduli space of (unrooted) phylogenetic trees with 7 vertices. The former was studied by Ardila and Klivans in [4, §4]. They develop the correspondence between ultrametrics and equidistant phylogenetic trees in [4, Theorem 3]. The latter is a tropicalization of the Grassmannian $\operatorname{Gr}(2,7)$ as described in [48, §4]. From the combinatorial description given there one derives the face numbers below:

Lemma 2.1. The tropical linear space trop $\left(\mathrm{Cyc}_{4}\right)$ is the space of ultrametrics on 6 elements, or, equivalently, the space of equidistant phylogenetic trees on 6 taxa. It is a fan over a threedimensional simplicial complex with 56 vertices, 490 edges, 1260 triangles and 945 tetrahedra.

We now define our two modular threefolds by way of a monomial map from $\mathbb{P}^{14}$ to another space $\mathbb{P}^{n}$. The homogeneous coordinates on that $\mathbb{P}^{n}$ will be denoted $m_{0}, m_{1}, \ldots, m_{n}$, so as to highlight that they can be identified with certain modular forms, known as theta constants.

The Segre cubic $\mathcal{S}$ is the closure of the image of $\mathrm{Cyc}_{4}$ under $\mathbb{P}_{-\rightarrow}^{14} \stackrel{\text { monomial }}{\mathbb{P}^{14}}$ given by

$$
\begin{gathered}
\left(z_{12} z_{34} z_{56}: z_{12} z_{35} z_{46}: z_{12} z_{36} z_{45}: z_{13} z_{24} z_{56}: z_{13} z_{25} z_{46}: z_{13} z_{26} z_{45}: z_{14} z_{23} z_{56}:\right. \\
\left.z_{14} z_{25} z_{36}: z_{14} z_{26} z_{35}: z_{15} z_{23} z_{46}: z_{15} z_{24} z_{36}: z_{15} z_{26} z_{34}: z_{16} z_{23} z_{45}: z_{16} z_{24} z_{35}: z_{16} z_{25} z_{34}\right) .
\end{gathered}
$$


The prime ideal of $\mathcal{S}$ is generated by 10 linear trinomials, like $m_{0}-m_{1}+m_{2}$, that come from Plücker relations among the $x_{i}-x_{j}$, and one cubic binomial such as $m_{0} m_{7} m_{12}-m_{2} m_{6} m_{14}$. For a graphical representation of this ideal we refer to Howard et al. [35, (1.2)]: for the connection, note that the monomials in (2.2) naturally correspond to perfect matchings of a set of size 6, which are the colored graphs in [35].

To see that this is the same as the classical definition of the Segre cubic, the reader can jump ahead to (2.8) and (2.9).

The Igusa quartic $\mathcal{I}$ is the closure of the image of $\mathrm{Cyc}_{4}$ under $\mathbb{P}^{14} \stackrel{\text { monomial }}{\rightarrow} \mathbb{P}^{9}$ given by

$\left(z_{12} z_{13} z_{23} z_{45} z_{46} z_{56}: z_{12} z_{14} z_{24} z_{35} z_{36} z_{56}: z_{12} z_{15} z_{25} z_{34} z_{36} z_{46}: z_{12} z_{16} z_{26} z_{34} z_{35} z_{45}: z_{13} z_{14} z_{34} z_{25} z_{26} z_{56}:\right.$

$\left.z_{13} z_{15} z_{35} z_{24} z_{26} z_{46}: z_{13} z_{16} z_{36} z_{24} z_{25} z_{45}: z_{14} z_{15} z_{45} z_{23} z_{26} z_{36}: z_{14} z_{16} z_{46} z_{23} z_{25} z_{35}: z_{15} z_{16} z_{56} z_{23} z_{24} z_{34}\right)$

The prime ideal of $\mathcal{I}$ is generated by the five linear forms in the column vector

$$
\left(\begin{array}{ccccc}
0 & m_{0} & m_{1} & m_{2} & m_{3} \\
m_{0} & 0 & m_{4} & m_{5} & m_{6} \\
m_{1} & m_{4} & 0 & m_{7} & m_{8} \\
m_{2} & m_{5} & m_{7} & 0 & m_{9} \\
m_{3} & m_{6} & m_{8} & m_{9} & 0
\end{array}\right) \cdot\left(\begin{array}{r}
1 \\
-1 \\
1 \\
-1 \\
1
\end{array}\right)
$$

together with any of the $4 \times 4$-minors of the symmetric $5 \times 5$-matrix in (2.3). The linear forms (2.3) come from Plücker relations of degree $(1,1,1,1,1,1)$ on $\operatorname{Gr}(3,6)$. We note that $m_{0}, \ldots, m_{9}$ can be written in terms of theta functions by Thomae's theorem [22, §VIII.5].

To see that this is the usual Igusa quartic, one can calculate the projective dual of the quartic hypersurface we have just described and verify that it is a cubic hypersurface whose singular locus consists of 10 nodes. The Segre cubic is the unique cubic in $\mathbb{P}^{4}$ with 10 nodes.

A key ingredient in the study of modular varieties is the symplectic combinatorics of finite vector spaces. Here we consider the binary space $\mathbb{F}_{2}^{4}$ with the symplectic form

$$
\langle x, y\rangle=x_{1} y_{3}+x_{2} y_{4}-x_{3} y_{1}-x_{4} y_{2} .
$$

We fix the following bijection between the 15 hyperplanes (2.1) and the vectors in $\mathbb{F}_{2}^{4} \backslash\{0\}$ :

$\begin{array}{ccccccccccccccc}z_{12} & z_{13} & z_{14} & z_{15} & z_{16} & z_{23} & z_{24} & z_{25} & z_{26} & z_{34} & z_{35} & z_{36} & z_{45} & z_{46} & z_{56} \\ u_{0001} & u_{1100} & u_{1110} & u_{0101} & u_{0110} & u_{1101} & u_{1111} & u_{0100} & u_{0111} & u_{0010} & u_{1001} & u_{1010} & u_{1011} & u_{1000} & u_{0011}\end{array}$

This bijection has the property that two vectors in $\mathbb{F}_{2}^{4} \backslash\{0\}$ are perpendicular with respect to (2.4) if and only if the corresponding elements of the root system $A_{5}$ are perpendicular. Combinatorially, this means that the two pairs of indices are disjoint. There are precisely 35 two-dimensional subspaces $L$ in $\mathbb{F}_{2}^{4}$. Of these planes $L$, precisely 15 are isotropic, which means that $L=L^{\perp}$. The other 20 planes naturally come in pairs $\left\{L, L^{\perp}\right\}$. Each plane is a triple in $\mathbb{F}_{2}^{4} \backslash\{0\}$ and we write it as a cubic monomial $z_{i j} z_{k \ell} z_{m n}$. Under this identification, the parametrization (2.2) of the Segre cubic $\mathcal{S}$ is given by the 15 isotropic planes $L$, while that of the Igusa quartic $\mathcal{I}$ is given by the 10 pairs $L \cdot L^{\perp}$ of non-isotropic planes in $\mathbb{F}_{2}^{4}$.

The symplectic group $\operatorname{Sp}_{4}\left(\mathbb{F}_{2}\right)$ consists of all linear automorphisms of $\mathbb{F}_{2}^{4}$ that preserve the symplectic form (2.4). As an abstract group it is isomorphic to the symmetric group on six letters:

$$
\operatorname{Sp}_{4}\left(\mathbb{F}_{2}\right) \cong \Sigma_{6}
$$


This group isomorphism is made explicit by the bijection (2.5).

Let $\mathcal{M}_{2}(2)$ denote the moduli space of smooth curves of genus 2 with a level 2 structure. In light of the isomorphism (2.6), a level 2 structure on a genus 2 curve $C$ is an ordering of its six Weierstrass points, and this corresponds to the choice of six labeled points on the projective line $\mathbb{P}^{1}$. The latter choices are parametrized by the moduli space $\mathcal{M}_{0,6}$. In what follows, we consider the open Segre cubic $\mathcal{S}^{\circ}=\mathcal{S} \backslash\left\{m_{0} m_{1} \cdots m_{14}=0\right\}$ inside the torus $\mathbb{G}_{m}^{14} \subset \mathbb{P}^{14}$ and the open Igusa quartic $\mathcal{I}^{\circ}=\mathcal{I} \backslash\left\{m_{0} m_{1} \cdots m_{9}=0\right\}$ inside the torus $\mathbb{G}_{m}^{9} \subset \mathbb{P}^{9}$.

Proposition 2.2. We have the following identification of three-dimensional moduli spaces:

$$
\mathcal{S}^{\circ}=\mathcal{I}^{\circ}=\mathcal{M}_{2}(2)=\mathcal{M}_{0,6}
$$

Proof. We already argued the last equation. The first equation is the isomorphism between the open sets $D$ and $D^{\prime}$ in the proof of [36, Theorem 3.3.11]. A nice way to see this isomorphism is that the kernels of our two monomial maps coincide (Lemma 2.3). The middle equation follows from the last part of [36, Theorem 3.3.8], which concerns the Kummer functor $\mathbf{K}_{2}$. For more information on the modular interpretations of $\mathcal{S}$ and $\mathcal{I}$ see $[22, \S \mathrm{VIII}]$.

The Kummer surface associated to a point in $\mathcal{I}^{\circ}$ is the intersection of the Igusa quartic $\mathcal{I}$ with the tangent space at that point, by [36, Theorem 3.3.8]. We find it convenient to express that Kummer surface in terms of the corresponding point in $\mathcal{S}^{\circ}$. Following Dolgachev and Ortland [22, §IX.5, Proposition 6], we write the defining equation of the Segre cubic $\mathcal{S}$ as

$$
16 r^{3}-4 r\left(s_{01}^{2}+s_{10}^{2}+s_{11}^{2}\right)+4 s_{01} s_{10} s_{11}+r t^{2}=0 .
$$

The embedding of the $\mathbb{P}^{4}$ with coordinates $\left(r: s_{01}: s_{10}: s_{11}: t\right)$ into our $\mathbb{P}^{14}$ can be written as

$$
\begin{aligned}
& r=m_{0}, \quad s_{01}=2 m_{0}-4 m_{1}, \quad s_{10}=2 m_{0}-4 m_{3}, \\
& s_{11}=4 m_{4}-2 m_{0}-4 m_{7}, \quad t=8\left(m_{1}+m_{3}-m_{0}-m_{4}-m_{7}\right) .
\end{aligned}
$$

This does not pick out an $\Sigma_{6}$-equivariant embedding of the space spanned by the $r, s_{i j}$ in the permutation representation of the $m_{i}$, but it has the advantage of giving short expressions. Fixing Schrödinger coordinates $\left(x_{00}: x_{01}: x_{10}: x_{11}\right)$ on $\mathbb{P}^{3}$, the Kummer surface is now given by

$$
\begin{gathered}
r\left(x_{00}^{4}+x_{01}^{4}+x_{10}^{4}+x_{11}^{4}\right)+s_{01}\left(x_{00}^{2} x_{01}^{2}+x_{10}^{2} x_{11}^{2}\right)+s_{10}\left(x_{00}^{2} x_{10}^{2}+x_{01}^{2} x_{11}^{2}\right) \\
+s_{11}\left(x_{00}^{2} x_{11}^{2}+x_{01}^{2} x_{10}^{2}\right)+t\left(x_{00} x_{01} x_{10} x_{11}\right)=0 .
\end{gathered}
$$

This equation is the determinant of the $5 \times 5$-matrix in [44, Example 1.1]. Its lower $4 \times 4$ minors satisfy (2.8). Our notation is consistent with that for the Coble quartic in [44, (2.13)].

We now come to the tropicalization of our three-dimensional moduli spaces. We write $e_{12}, e_{13}, \ldots, e_{56}$ for the unit vectors in $\mathbb{T P}^{14}=\mathbb{R}^{15} / \mathbb{R}(1,1, \ldots, 1)$. These correspond to our coordinates $z_{12}, z_{13}, \ldots, z_{56}$ on the $\mathbb{P}^{14}$ which contains $\mathrm{Cyc}_{4} \simeq \mathbb{P}^{4}$. The 56 rays of the Bergman fan $\operatorname{trop}\left(\mathrm{Cyc}_{4}\right)$ are indexed by proper subsets $\sigma \varsubsetneqq\{1,2,3,4,5,6\}$ with $|\sigma| \geq 2$. They are

$$
E_{\sigma}=\sum_{\{i, j\} \subseteq \sigma} e_{i j} .
$$

Cones in trop $\left(\mathrm{Cyc}_{4}\right)$ are spanned by collections of $E_{\sigma}$ whose indices $\sigma$ are nested or disjoint. 
Let $A_{\text {segre }}$ denote the $15 \times 15$-matrix with entries in $\{0,1\}$ that represents the tropicalization of the monomial map (2.2). The columns of $A_{\text {segre }}$ are indexed by (2.5). The rows of $A_{\text {segre }}$ are indexed by tripartitions of $\{1,2, \ldots, 6\}$, or by isotropic planes in $\mathbb{F}_{2}^{4}$. An entry is 1 if the pair that indexes the column appears in the tripartition that indexes the row, or, equivalently, if the line of $\mathbb{F}_{2}^{4}$ that indexes the column is contained in the plane that indexes the row. Note that each row and each column of $A_{\text {segre }}$ has precisely three nonzero entries.

We similarly define the $10 \times 15$-matrix $A_{\text {igusa }}$ with entries in $\{0,1\}$ that represents the monomial map for the Igusa quartic. Its rows have six nonzero entries and its columns have four nonzero entries. The column labels of $A_{\text {igusa }}$ are the same as those of $A_{\text {segre }}$. The rows are now labeled by bipartitions of $\{1,2, \ldots, 6\}$, or by pairs of non-isotropic planes in $\mathbb{F}_{2}^{4}$.

Lemma 2.3. The matrices $A_{\text {segre }}$ and $A_{\text {igusa }}$ have the same kernel. This kernel is the 5dimensional subspace spanned by the vectors $E_{\sigma}-E_{\sigma^{c}}$ where $\sigma$ runs over triples in $\{1,2, \ldots, 6\}$.

This lemma can be proved by a direct computation. The multiplicative version of this fact implies the identity $\mathcal{S}^{\circ}=\mathcal{I}^{\circ}$ as seen in Proposition 2.2. We have the following result.

Theorem 2.4. The tropical Segre cubic $\operatorname{trop}(\mathcal{S})$ in $\mathbb{T P}^{14}$ is the image of trop $\left(\mathrm{Cyc}_{4}\right)$ under the linear map $A_{\text {segre. }}$ The tropical Igusa quartic $\operatorname{trop}(\mathcal{I})$ in $\mathbb{T P}^{9}$ is the image of trop $\left(\mathrm{Cyc}_{4}\right)$ under the linear map $A_{\text {igusa. }}$. These two 3-dimensional fans are affinely isomorphic to each other, but all maximal cones of $\operatorname{trop}(\mathcal{I})$ come with multiplicity two. The underlying simplicial complex has 25 vertices, 105 edges and 105 triangles. This is the tree space $\operatorname{trop}\left(\mathcal{M}_{0,6}\right)$.

Proof. The fact that we can compute the tropicalization of the image of a linear space under a monomial map by just applying the tropicalized monomial map $A$ • to the Bergman fan is [20, Theorem 3.1]. The fact that the two tropical threefolds are affinely isomorphic follows immediately from Lemma 2.3. To analyze the combinatorics of this common image fan, we set $E_{\sigma}$ to be the zero vector when $\sigma=\{i\}$ is a singleton. With this convention, we have

$$
A_{\text {segre }} E_{\sigma}=A_{\text {segre }} E_{\sigma^{c}} \text { and } A_{\text {igusa }} E_{\sigma}=A_{\text {igusa }} E_{\sigma^{c}}
$$

for all proper subsets $\sigma$ of $\{1,2, \ldots, 6\}$. We conclude that the $56=15+20+15+6$ rays of the Bergman fan trop $\left(\mathrm{Cyc}_{4}\right)$ get mapped to $25=15+10$ distinct rays in the image fan.

The cones in trop $\left(\mathrm{Cyc}_{4}\right)$ correspond to equidistant trees, that is, rooted metric trees on six taxa. Combinatorially, our map corresponds to removing the root from the tree, so the cones in the image fan correspond to unrooted metric trees on six taxa. Specifically, each of the 945 maximal cones of $\operatorname{trop}\left(\mathrm{Cyc}_{4}\right)$ either has one ray $E_{\{i, j, k, \ell, m\}}$ that gets mapped to zero, or it has two rays $E_{\sigma}$ and $E_{\sigma^{c}}$ that become identified. Therefore its image is threedimensional. Our map takes the 945 simplicial cones of dimension 4 in trop $\left(\mathrm{Cyc}_{4}\right)$ onto the 105 simplicial cones of dimension 3, one for each unrooted tree. The fibers involve precisely nine cones because each trivalent tree on six taxa has nine edges, each a potential root location. Combinatorially, nine rooted trivalent trees map to the same unrooted tree.

It remains to analyze the multiplicity of each maximal cone in the image. The 105 maximal cones in $\operatorname{trop}(\mathcal{S})$ all have multiplicity one, while the corresponding cones in $\operatorname{trop}(\mathcal{I})$ have multiplicity two. We first found this by a direct calculation using the software gfan [37], starting from the homogeneous ideals of $\mathcal{S}$ and $\mathcal{I}$ described above. It can also be seen by examining the images of the rays $E_{\tau}$ under each matrix $A_{\bullet}$ modulo the line spanned by 
the vector $(1,1, \ldots, 1)$. Each of the 15 vectors $A_{\text {igusa }} E_{i j}$ is the sum of four unit vectors in $\mathbb{T P}^{9}$, while the 10 vectors $A_{\text {igusa }} E_{i j k}$ are the ten unit vectors multiplied by the factor 2.

We next discuss the tropicalization of the universal family of Kummer surfaces over $\mathcal{S}^{\circ}$. This is the hypersurface in $\mathcal{S}^{\circ} \times \mathbb{P}^{3}$ defined by the equation (2.10). The tropicalization of this hypersurface is a five-dimensional fan whose fibers over the tree space $\operatorname{trop}(\mathcal{S})$ are the tropical Kummer surfaces in $\mathbb{T P}^{3}$. We computed this fan from the equations using gfan [37].

Proposition 2.5. The tropicalization of the universal Kummer surface in the coordinates $\left(\left(m_{0}: m_{1}: \cdots: m_{14}\right),\left(x_{00}: x_{01}: x_{10}: x_{11}\right)\right)$ is a 5-dimensional polyhedral fan in $\mathbb{T P}^{14} \times \mathbb{T P}^{3}$. This fan has 56 rays and 1536 maximal cones, and its f-vector is (56, 499, 1738, 2685, 1536).

Instances of tropical Kummer surfaces can be obtained by slicing the above fan with fixed values of the 15 tropical $m$ coordinates. Figure 3 shows the tropicalization of a Kummer surface over a snowflake tree (Type (7) in Table 3). It consists of 30 two-dimensional polyhedra, 24 unbounded and 6 bounded. The latter 6 form the facets of a parallelepiped.

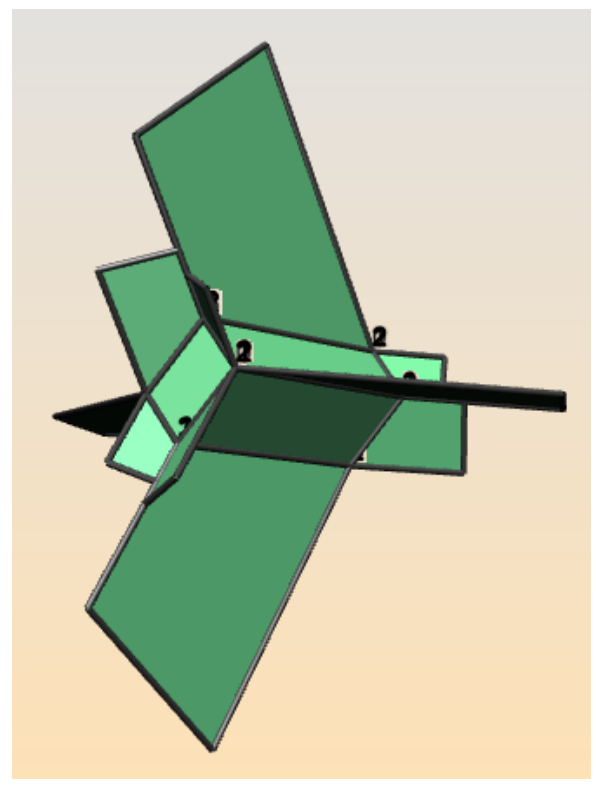

Figure 3: Tropicalization of a Kummer surface over a snowflake tree.

Figure 4 shows a tropical Kummer surface over a caterpillar tree (Type (6) in Table 3). It consists of 33 two-dimensional polyhedra, 24 bounded and 9 bounded. The latter 9 polygons form a subdivision of a flat octagon. These two pictures were drawn using polymake [28].

On each Kummer surface we could now identify a tree that represents the bicanonical image of the associated genus 2 curve. Classically, one obtains a double quadric with six distinguished points by intersecting with any of the planes in the $16_{6}$ configuration [44, (1.2)].

The tropical variety described in Theorem 2.4 defines the tropical compactification $\overline{\mathcal{S}}$ of the Segre cubic $\mathcal{S}$. By definition, the threefold $\overline{\mathcal{S}}$ is the closure of $\mathcal{S}^{\circ}$ in the toric variety determined by the given fan structure on $\operatorname{trop}(\mathcal{S})$. For details, see Tevelev's article [50].

This tropical compactification of our moduli space (2.7) is intrinsic. To see this, we recall that the intrinsic torus of a very affine variety $X \subset \mathbb{G}_{m}^{n}$ is the torus whose character lattice 


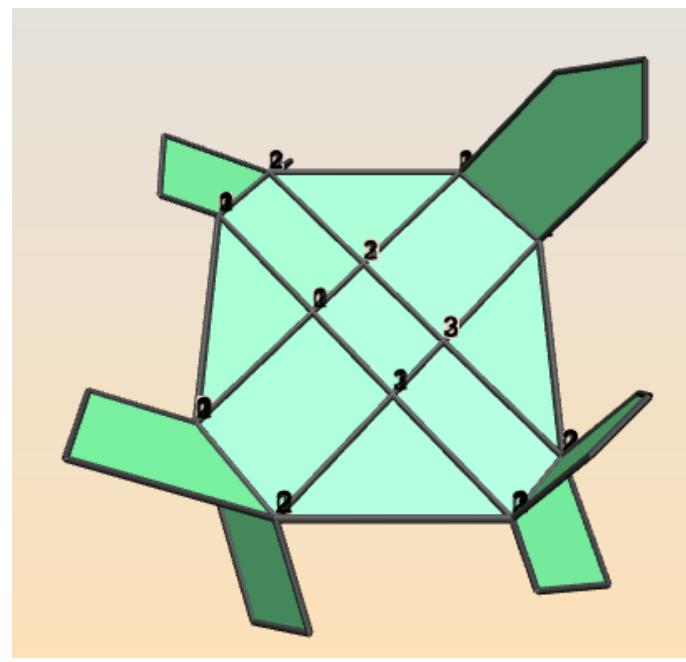

Figure 4: Tropicalization of a Kummer surface over a caterpillar tree.

is the finitely generated multiplicative free abelian group $K[X]^{*} / K^{*}$. The following lemma can be used to find the intrinsic torus for each of the very affine varieties in this paper.

Lemma 2.6. Let $m: T_{1} \rightarrow \underline{T_{2}}$ be a monomial map of tori and $U \subset T_{1}$ a subvariety embedded in its intrinsic torus. Then $\overline{m(U)} \subset m\left(T_{1}\right)$ is the embedding of $\overline{m(U)}$ in its intrinsic torus.

Proof. Choose identifications $K\left[T_{1}\right]=K\left[x_{1}^{ \pm}, \ldots, x_{r}^{ \pm}\right]$and $K\left[T_{2}\right]=K\left[y_{1}^{ \pm}, \ldots, y_{s}^{ \pm}\right]$. By assumption, the pullback $m^{*}\left(y_{i}\right)$ is a monomial in the $x_{j}$, which we call $z_{i}$. We have an injection of rings $m^{*}: K[\overline{m(U)}] \subset K[U]$, and hence we get an induced injection of groups $\phi: K[\overline{m(U)}]^{*} / K^{*} \subset K[U]^{*} / K^{*}$. Since $K[\overline{m(U)}]$ is generated by the $y_{i}$, we conclude that $m^{*}(K[\overline{m(U)}])$ is contained in the subalgebra $K\left[z_{1}^{ \pm}, \ldots, z_{s}^{ \pm}\right] \subset K[U]$. Pick $f \in K[\overline{m(U)}]^{*} / K^{*}$. Since $U$ is embedded in its intrinsic torus, we have $\phi(f)=z_{1}^{d_{1}} \cdots z_{s}^{d_{s}}$ for some $d_{i} \in \mathbb{Z}$. So $\phi\left(y_{1}^{d_{1}} \cdots y_{s}^{d_{s}}\right)=\phi(f)$ and since $\phi$ is injective, we conclude that $f=y_{1}^{d_{1}} \cdots y_{s}^{d_{s}}$.

The embedding of the Segre cubic $\mathcal{S}$ into the 9-dimensional toric variety given by (2.2) satisfies the hypotheses of Lemma 2.6. Indeed, $\mathcal{S}^{\circ}$ is the image of the complement of a hyperplane arrangement under a monomial map, and, by [50, §4], the intrinsic torus of an essential arrangement of $n$ hyperplanes in $\mathbb{P}^{r}$ is $\mathbb{G}_{m}^{n-1}$. The same argument works for all moduli spaces studied in this paper. That the ambient torus $\mathbb{G}_{m}^{9}$ is intrinsic for the open Segre cubic $\mathcal{S}^{\circ}$ can also be seen from the fact that the 15 boundary divisors $\mathcal{S} \cap\left\{m_{i}=0\right\}$ are irreducible. Indeed, by $[36, \S 3.2 .1]$, they are projective planes $\mathbb{P}^{2}$. Each of the ten singular points of $\mathcal{S}$ lies on six of these planes, so each boundary plane contains four singular points.

From the combinatorial description above we infer the following summary of the situation.

Corollary 2.7. The tropical compactification of the open Segre cubic $\mathcal{S}^{\circ}$, and hence of the other moduli spaces in (2.7), is the Deligne-Mumford compactification $\overline{\mathcal{M}}_{0,6}$. This threefold is the blow-up of the 10 singular points of $\mathcal{S}$, or of the 15 singular lines of the Igusa quartic $\mathcal{I}$.

The second sentence is Theorem 3.3.11 in Hunt's book [36]. The first is a special case of [33, Theorem 1.11]. Our rationale for giving a detailed equational derivation of the familiar manifold $\overline{\mathcal{M}}_{0,6}$ is that it sets the stage for our primary example in the next section. 


\section{Burkhardt Quartic and Abelian Surfaces}

The Burkhardt quartic is a rational quartic threefold in $\mathbb{P}^{4}$. It can be characterized as the unique quartic hypersurface in $\mathbb{P}^{4}$ with the maximal number 45 of nodal singular points [19]. It compactifies the moduli space $\mathcal{M}_{2}(3)$ of genus 2 curves with level 3 structure [21, 27, 29, 36]. We identify $\mathcal{M}_{2}(3)$ with a subvariety of $\mathcal{A}_{2}(3)$, the moduli space of principally polarized abelian surfaces with level 3 structure, by sending a smooth curve to its Jacobian.

All constructions in this section can be carried out over any field $K$ of characteristic other than 2 or 3, provided $K$ contains a primitive third root of unity $\omega$. In the tropical context, $K$ will be a field with a valuation. For details on arithmetic issues see Elkies' paper [23].

We realize the Burkhardt quartic as the image of a rational map that is given as a composition $\mathbb{P}^{3} \stackrel{\text { linear }}{\hookrightarrow} \mathbb{P}^{39} \stackrel{\substack{\text { monomial } \\-\rightarrow}}{\mathbb{P}^{39}}$. We choose coordinates $\left(c_{0}: c_{1}: c_{2}: c_{3}\right)$ on $\mathbb{P}^{3}$ and coordinates $\left(m_{0}: m_{1}: \cdots: m_{39}\right)$ on the rightmost $\mathbb{P}^{39}$. The 40 homogeneous coordinates $u_{i j k \ell}$ on the middle $\mathbb{P}^{39}$ are indexed by the lines through the origin in the finite vector space $\mathbb{F}_{3}^{4}$. Each line is given by the vector whose leftmost nonzero coordinate is 1 . The linear map $\mathbb{P}^{3} \hookrightarrow \mathbb{P}^{39}$ is defined as follows, where $\omega=\frac{1}{2}(-1+\sqrt{-3})$ is a third root of unity:

$$
\begin{array}{cccc}
u_{0001}=c_{1}+c_{2}+c_{3} & u_{0010}=c_{2}-c_{3}+c_{0} & u_{0011}=c_{3}+c_{0}-c_{1} & u_{0012}=c_{0}+c_{1}-c_{2} \\
u_{0100}=\sqrt{-3} \cdot c_{1} & u_{0101}=c_{1}+\omega^{2} c_{2}+\omega^{2} c_{3} & u_{0102}=c_{1}+\omega c_{2}+\omega c_{3} & u_{0110}=c_{2}-\omega c_{3}+\omega^{2} c_{0} \\
u_{0111}=c_{3}+c_{0}-\omega c_{1} & u_{0112}=c_{0}+\omega^{2} c_{1}-c_{2} & u_{0120}=c_{2}-\omega^{2} c_{3}+\omega c_{0} & u_{0121}=c_{0}+\omega c_{1}-c_{2} \\
u_{0122}=c_{3}+c_{0}-\omega^{2} c_{1} & u_{1000}=\sqrt{-3} \cdot c_{0} & u_{1001}=c_{1}+\omega c_{2}+\omega^{2} c_{3} & u_{1002}=c_{1}+\omega^{2} c_{2}+\omega c_{3} \\
u_{1010}=c_{2}-c_{3}+\omega c_{0} & u_{1011}=c_{3}+\omega c_{0}-c_{1} & u_{1012}=c_{0}+\omega^{2} c_{1}-\omega^{2} c_{2} & u_{1020}=c_{2}-c_{3}+\omega^{2} c_{0} \\
u_{1021}=c_{0}+c_{1} \omega-\omega c_{2} & u_{1022}=c_{3}+\omega^{2} c_{0}-c_{1} & u_{1100}=\sqrt{-3} \cdot c_{3} & u_{1101}=c_{1}+c_{2}+\omega c_{3} \\
u_{1102}=c_{1}+c_{2}+c_{3} \omega^{2} & u_{1110}=c_{2}-\omega c_{3}+c_{0} & u_{1111}=c_{3}+\omega c_{0}-\omega c_{1} & u_{1112}=c_{0}+\omega c_{1}-\omega^{2} c_{2} \\
u_{1120}=c_{2}-\omega^{2} c_{3}+c_{0} & u_{1121}=c_{0}+\omega^{2} c_{1}-\omega c_{2} & u_{1122}=c_{3}+c_{0} \omega^{2}-\omega^{2} c_{1} & u_{1200}=\sqrt{-3} \cdot c_{2} \\
u_{1201}=c_{1}+\omega^{2} c_{2}+c_{3} & u_{1202}=c_{1}+\omega c_{2}+c_{3} & u_{1210}=c_{2}-\omega^{2} c_{3}+\omega^{2} c_{0} & u_{1211}=c_{3}+\omega c_{0}-\omega^{2} c_{1} \\
u_{1212}=c_{0}+c_{1}-\omega^{2} c_{2} & u_{1220}=c_{2}-\omega c_{3}+\omega c_{0} & u_{1221}=c_{0}+c_{1}-\omega c_{2} & u_{1222}=c_{3}+\omega^{2} c_{0}-\omega c_{1}
\end{array}
$$

These 40 linear forms cut out the hyperplanes of the complex reflection arrangement $\mathrm{G}_{32}$. We refer to the book by Hunt $[36, \S 5]$ for a discussion of this arrangement and its importance for modular Siegel threefolds. Our first map $\mathbb{P}^{3} \hookrightarrow \mathbb{P}^{39}$ realizes the arrangement $\mathrm{G}_{32}$ as the restriction of the 40 coordinate planes in $\mathbb{P}^{39}$ to a certain 3-dimensional linear subspace.

The monomial map $\mathbb{P}^{39} \rightarrow \mathbb{P}^{39}$ is defined outside the hyperplane arrangement $\left\{\prod u_{i j k \ell}=\right.$ 0 \} which corresponds to $\mathrm{G}_{32}$. It is given by the following 40 monomials of degree four:

$$
\begin{array}{lll}
m_{0}=u_{0001} u_{0010} u_{0011} u_{0012} & m_{1}=u_{0001} u_{1000} u_{1001} u_{1002} & m_{2}=u_{0001} u_{1010} u_{1011} u_{1012} \\
m_{3}=u_{0001} u_{1020} u_{1021} u_{1022} & m_{4}=u_{0010} u_{0100} u_{0110} u_{0120} & m_{5}=u_{0010} u_{0101} u_{0111} u_{0121} \\
m_{6}=u_{0010} u_{0102} u_{0112} u_{0122} & m_{7}=u_{0011} u_{1200} u_{1211} u_{1222} & m_{8}=u_{0011} u_{1201} u_{1212} u_{1220} \\
m_{9}=u_{0011} u_{1202} u_{1210} u_{1221} & m_{10}=u_{0012} u_{1100} u_{1112} u_{1121} & m_{11}=u_{0012} u_{1101} u_{1110} u_{1122} \\
m_{12}=u_{0012} u_{1102} u_{1111} u_{1120} & m_{13}=u_{0100} u_{1000} u_{1100} u_{1200} & m_{14}=u_{0100} u_{1010} u_{1110} u_{1210} \\
m_{15}=u_{0100} u_{1020} u_{1120} u_{1220} & m_{16}=u_{0101} u_{1000} u_{1101} u_{1202} & m_{17}=u_{0101} u_{1010} u_{1111} u_{1212} \\
m_{18}=u_{0101} u_{1020} u_{1121} u_{1222} & m_{19}=u_{0102} u_{1000} u_{1102} u_{1201} & m_{20}=u_{0102} u_{1010} u_{1112} u_{1211} \\
m_{21}=u_{0102} u_{1020} u_{1122} u_{1221} & m_{22}=u_{0110} u_{1001} u_{1111} u_{1221} & m_{23}=u_{0110} u_{1011} u_{1121} u_{1201} \\
m_{24}=u_{0110} u_{1021} u_{1101} u_{1211} & m_{25}=u_{0111} u_{1001} u_{1112} u_{1220} & m_{26}=u_{0111} u_{1011} u_{1122} u_{1200} \\
m_{27}=u_{0111} u_{1021} u_{1102} u_{1210} & m_{28}=u_{0112} u_{1001} u_{1110} u_{1222} & m_{29}=u_{0112} u_{1011} u_{1120} u_{1202}
\end{array}
$$




$$
\begin{array}{lll}
m_{30}=u_{0112} u_{1021} u_{1100} u_{1212} & m_{31}=u_{0120} u_{1002} u_{1122} u_{1212} & m_{32}=u_{0120} u_{1012} u_{1102} u_{1222} \\
m_{33}=u_{0120} u_{1022} u_{1112} u_{1202} & m_{34}=u_{0121} u_{1002} u_{1120} u_{1211} & m_{35}=u_{0121} u_{1012} u_{1100} u_{1221} \\
m_{36}=u_{0121} u_{1022} u_{1110} u_{1201} & m_{37}=u_{0122} u_{1002} u_{1121} u_{1210} & m_{38}=u_{0122} u_{1012} u_{1101} u_{1220} \\
m_{39}=u_{0122} u_{1022} u_{1111} u_{1200} . & &
\end{array}
$$

The combinatorics behind this list is as follows. The 40 monomials represent the 40 isotropic planes in the space $\mathbb{F}_{3}^{4}$, with respect to the symplectic inner product (2.4). The linear inclusion $\mathbb{P}^{3} \hookrightarrow \mathbb{P}^{39}$ has the property that two linearly independent vectors $x, y$ in $\mathbb{F}_{3}^{4}$ satisfy $\langle x, y\rangle=0$ if and only if the corresponding linear forms $u_{x}$ and $u_{y}$ are perpendicular in the root system $\mathrm{G}_{32}$, using the usual Hermitian inner product (when considered over $\mathbb{C}$ ).

Let $\mathcal{B}$ denote the Burkhardt quartic in $\mathbb{P}^{39}$, that is, the closure of the image of the map above. Its homogeneous prime ideal $I_{\mathcal{B}}$ is minimally generated by one quartic and a 35 dimensional space of linear forms in $K\left[m_{0}, m_{1}, \ldots, m_{39}\right]$. That space has a natural generating set consisting of $160=4 \cdot 40$ linear trinomials. Namely, the four coordinates $m_{\bullet}$ that share a common parameter $u_{i j k \ell}$ span a two-dimensional space modulo $I_{\mathcal{B}}$. For instance, the first four coordinates share the parameter $u_{0001}$, and they satisfy the following linear trinomials:

$$
\begin{aligned}
& m_{0}+\omega^{2} m_{1}-\omega m_{2}=m_{0}-\omega m_{1}-\omega^{2} m_{3}= \\
& m_{0}+\omega^{2} m_{2}+\omega m_{3}=m_{1}+\omega m_{2}-\omega^{2} m_{3}=0 .
\end{aligned}
$$

These relations are constructed as follows: Each of the 40 roots $u_{i j k \ell}$ appears as a factor in precisely four of the coordinates $m_{\bullet}$, and these four span a two-dimensional space over $K$.

The 160 linear trinomials (3.1) cut out a 4-dimensional linear subspace of $\mathbb{P}^{39}$. We fix the following system of coordinates, analogous to $(2.9)$, on that linear subspace $\mathbb{P}^{4}$ of $\mathbb{P}^{39}$ :

$$
\begin{array}{cccc}
r= & 3 c_{0} c_{1} c_{2} c_{3} & = & m_{13} / 3 \\
s_{01}= & -c_{0}\left(c_{1}^{3}+c_{2}^{3}+c_{3}^{3}\right) & = & \left(\sqrt{-3} \cdot m_{1}-m_{13}\right) / 3 \\
s_{10}= & c_{1}\left(c_{0}^{3}+c_{2}^{3}-c_{3}^{3}\right) & = & \left(-\sqrt{-3} \cdot m_{4}-m_{13}\right) / 3 \\
s_{11}= & c_{2}\left(c_{0}^{3}-c_{1}^{3}+c_{3}^{3}\right) & = & \left(-\sqrt{-3} \cdot m_{7}-m_{13}\right) / 3 \\
s_{12}= & c_{3}\left(c_{0}^{3}+c_{1}^{3}-c_{2}^{3}\right) & = & \left(-\sqrt{-3} \cdot m_{10}-m_{13}\right) / 3
\end{array}
$$

The polynomial that defines the Burkhardt quartic $\mathcal{B} \subset \mathbb{P}^{4}$ is now written as

$$
r\left(r^{3}+s_{01}^{3}+s_{10}^{3}+s_{11}^{3}+s_{12}^{3}\right)+3 s_{01} s_{10} s_{11} s_{12}=0 .
$$

The Burkhardt quartic has 45 isolated singular points. For example, one of the singular points is $\left(r: s_{01}: s_{10}: s_{11}: s_{12}\right)=(0: 0: 0: 1: 1)$. In the $m$-coordinates, this point is

$$
\begin{gathered}
\left(0: 0: 0: 0: 0: 0: 0:-\omega^{2}:-\omega: 1: \omega^{2}:-1: \omega: 0: 0: 0: 0: 0:\right. \\
0: 0: 0: 0:-\omega^{2}:-1:-\omega:-1:-\omega^{2}: \omega^{2}:-\omega: \omega^{2}: \\
\left.\omega^{2}: \omega^{2}: 1: \omega: 1: \omega^{2}:-\omega^{2}: \omega:-\omega^{2}:-\omega^{2}\right)
\end{gathered}
$$

For each singular point precisely 16 of the $40 \mathrm{~m}$-coordinates are zero. Each hyperplane $m_{\bullet}=0$ intersects the Burkhardt quartic $\mathcal{B}$ in a tetrahedron of four planes, known as Jacobi planes, which contains 18 of the 45 singular points, in a configuration that is depicted in [36, Figure 5.3(b)]. The relevant combinatorics will be explained when tropicalizing in Section 4.

The closure of the image of the monomial map $\mathbb{P}^{39} \rightarrow \mathbb{P}^{39}, u \mapsto m$ is a toric variety $\mathcal{T}$. Writing $\mathbb{P}^{4}$ for the linear subspace defined by the 160 trinomials like (3.1), we have

$$
\mathcal{B}=\mathcal{T} \cap \mathbb{P}^{4} \subset \mathbb{P}^{39} .
$$


Thus we have realized the Burkhardt quartic as a linear section of the toric variety $\mathcal{T}$, and it makes sense to explore the combinatorial properties of $\mathcal{T}$. Let $A$ denote the $40 \times 40$ matrix representing our monomial map $u \mapsto m$. The columns of $A$ are indexed by the $u_{i j k \ell}$, and hence by the lines in $\mathbb{F}_{3}^{4}$. The rows of $A$ are indexed by the $m_{\bullet}$, and hence by the isotropic planes in $\mathbb{F}_{3}^{4}$. The matrix $A$ is the 0 - 1 matrix that encodes incidences of lines and isotropic planes. Each row and each column has exactly four entries 1, and the other entries are 0. The matrix $A$ has rank 25, and we computed its Markov basis using the software 4ti2 [1].

Proposition 3.1. (a) The projective toric variety $\mathcal{T}$ has dimension 24.

(b) Its prime ideal is minimally generated by 5136 binomials, namely 216 binomials of degree 5, 270 of degree 6, 4410 of degree 8, and 240 of degree 12.

(c) The Burkhardt quartic is the scheme-theoretic intersection in (3.5). This intersection is not ideal-theoretic, since there is no quartic relation on $\mathcal{T}$ that could specialize to (3.3).

(d) The 24-dimensional polytope of $\mathcal{T}$, which is the convex hull of the 40 rows of A, has precisely 13144 facets.

Proof. (a) follows from the fact that $\operatorname{rank}(A)=25$. The statements in (b) and (c) follow from our 4ti2 calculation. The facets in (d) were computed using the software polymake [28]. The scheme-theoretic intersection in (c) can be verified by taking the following five among the 216 quintic binomials that vanish on $\mathcal{T}$ :

$$
\begin{array}{cc}
m_{0} m_{13} m_{22} m_{33} m_{37}-m_{1} m_{4} m_{9} m_{10} m_{39} & m_{0} m_{14} m_{23} m_{33} m_{35}-m_{2} m_{4} m_{9} m_{10} m_{36} \\
m_{0} m_{16} m_{25} m_{35} m_{37}-m_{1} m_{5} m_{9} m_{10} m_{38} & m_{0} m_{17} m_{26} m_{36} m_{38}-m_{2} m_{5} m_{8} m_{11} m_{39} \\
m_{9} m_{11} m_{13} m_{18} m_{20}-m_{7} m_{10} m_{14} m_{16} m_{21} &
\end{array}
$$

Each of these quintic binomials factors on $\mathbb{P}^{4}$ as the Burkhardt quartic (3.5) times a linear form, and these five linear forms generate the irrelevant maximal ideal $\left\langle r, s_{01}, s_{10}, s_{11}, s_{12}\right\rangle$.

We next explain the connection to abelian surfaces. Consider the open Burkhardt quartic

$$
\mathcal{B}^{\circ}=\mathcal{B} \backslash\left\{\prod m_{i}=0\right\} \subset \mathbb{P}^{39} .
$$

In its modular interpretation ([27], [29, §3.1], [36, Lemma 5.7.1]), this threefold is the moduli space $\mathcal{M}_{2}(3)$ of smooth genus 2 curves with level 3 structure. With every point $\left(r: s_{01}: s_{10}\right.$ : $\left.s_{11}: s_{12}\right) \in \mathcal{B}^{\circ}$ we associate an abelian surface (which is a Jacobian) following [29, §3.2]. The ambient space for this family of abelian surfaces is the projective space $\mathbb{P}^{8}$ whose coordinates

$$
\left(x_{00}: x_{01}: x_{02}: x_{10}: x_{11}: x_{12}: x_{20}: x_{21}: x_{22}\right)
$$

are indexed by $\mathbb{F}_{3}^{2}$. The following five polynomials represent all the affine subspaces of $\mathbb{F}_{3}^{2}$ :

$$
\begin{aligned}
f & =x_{00}^{3}+x_{01}^{3}+x_{02}^{3}+x_{10}^{3}+x_{11}^{3}+x_{12}^{3}+x_{20}^{3}+x_{21}^{3}+x_{22}^{3}, \\
g_{01} & =3\left(x_{00} x_{01} x_{02}+x_{10} x_{11} x_{12}+x_{20} x_{21} x_{22}\right), \\
g_{10} & =3\left(x_{00} x_{10} x_{20}+x_{01} x_{11} x_{21}+x_{02} x_{12} x_{22}\right), \\
g_{11} & =3\left(x_{00} x_{11} x_{22}+x_{01} x_{12} x_{20}+x_{10} x_{21} x_{02}\right), \\
g_{12} & =3\left(x_{00} x_{12} x_{21}+x_{01} x_{10} x_{22}+x_{02} x_{11} x_{20}\right) .
\end{aligned}
$$

Our abelian surface is the singular locus of the Coble cubic $\{C=0\}$ in $\mathbb{P}^{8}$, which is given by

$$
C=r f+s_{01} g_{01}+s_{10} g_{10}+s_{11} g_{11}+s_{12} g_{12} \text {. }
$$


Theorem 3.2. The singular locus of the Coble cubic of any point in $\mathcal{B}^{\circ}$ is an abelian surface $S$ of degree 18 in $\mathbb{P}^{8}$. This equips $S$ with an indecomposable polarization of type $(3,3)$. The prime ideal of $S$ is minimally generated by 9 quadrics and 3 cubics. The theta divisor on $S$ is a tricanonical curve of genus 2 , and this is obtained by intersecting $S$ with the $\mathbb{P}^{4}$ defined by

$$
\operatorname{rank}\left(\begin{array}{ccccc}
x_{00} & x_{01}+x_{02} & x_{10}+x_{20} & x_{11}+x_{22} & x_{12}+x_{21} \\
r & s_{01} & s_{10} & s_{11} & s_{12}
\end{array}\right) \leq 1
$$

Proof. The first statement is classical (see [8, §10.7]). We shall explain it below using theta functions. The fact about ideal generators is due to Gunji [31, Theorem 8.3]. The representation (3.6) of the curve whose Jacobian is $S$ is derived from [29, Theorem 3.14(d)].

We now discuss the complex analytic view of our story. Recall (e.g. from [8, §8.1]) that a principally polarized abelian surface over $\mathbb{C}$ is given analytically as $S_{\tau}=\mathbb{C}^{2} /\left(\mathbb{Z}^{2}+\tau \mathbb{Z}^{2}\right)$, where $\tau$ is a complex symmetric $2 \times 2$-matrix whose imaginary part is positive definite. The set of such matrices is the Siegel upper half space $\mathfrak{H}_{2}$. Fix the $4 \times 4$ matrix $J=\left[\begin{array}{cc}0 & -\operatorname{Id}_{2} \\ \operatorname{Id}_{2} & 0\end{array}\right]$. Let $\mathrm{Sp}_{4}(\mathbb{Z})$ be the group of $4 \times 4$ integer-valued matrices $\gamma$ such that $\gamma J \gamma^{T}=J$. This acts on $\mathfrak{H}_{2}$ via

$$
\left[\begin{array}{ll}
A & B \\
C & D
\end{array}\right] \cdot \tau=(A \tau+B)(C \tau+D)^{-1}
$$

where $A, B, C, D$ are $2 \times 2$ matrices, and this descends to an action of $\operatorname{PSp}_{4}(\mathbb{Z})$ on $\mathfrak{H}_{2}$. The natural map $\mathrm{PSp}_{4}(\mathbb{Z}) \rightarrow \mathrm{PSp}_{4}\left(\mathbb{F}_{3}\right)$ takes the residue class modulo 3 of each matrix entry. Let $\Gamma_{2}(3)$ denote the kernel of this map. The action of $\operatorname{PSp}_{4}(\mathbb{Z})$ preserves the abelian surface, while $\Gamma_{2}(3)$ preserves the abelian surface together with a level 3 structure. Hence $\mathfrak{H}_{2} / \mathrm{PSp}_{4}(\mathbb{Z})$ is the moduli space $\mathcal{A}_{2}$ of principally polarized abelian surfaces, while $\mathfrak{H}_{2} / \Gamma_{2}(3)$ is the moduli space $\mathcal{A}_{2}(3)$ of principally polarized abelian surfaces with level 3 structure. The finite group $\mathrm{PSp}_{4}\left(\mathbb{F}_{3}\right)$ is a simple group of order 25920 and it acts naturally on $\mathfrak{H}_{2} / \Gamma_{2}(3)$.

The third-order theta function with characteristic $\sigma \in \frac{1}{3} \mathbb{Z}^{2} / \mathbb{Z}^{2}$ is defined as

$$
\begin{aligned}
\Theta_{3}[\sigma](\tau, z) & =\exp \left(3 \pi i \sigma^{T} \tau \sigma+6 \pi i \sigma^{T} z\right) \cdot \theta(3 \tau, 3 z+3 \tau \sigma) \\
& =\sum_{n \in \mathbb{Z}^{2}} \exp \left(3 \pi i(n+\sigma)^{T} \tau(n+\sigma)+6 \pi i(n+\sigma)^{T} z\right) .
\end{aligned}
$$

Here $\theta$ is the classical Riemann theta function. For a fixed matrix $\tau \in \mathfrak{H}_{2}$, the nine third-order theta functions on $\mathbb{C}^{2}$ give precisely our embedding of the abelian surface $S_{\tau}$ into $\mathbb{P}^{8}$ :

$$
S_{\tau} \hookrightarrow \mathbb{P}^{8}, \quad z \mapsto\left(\Theta_{3}[\sigma](\tau, z)\right)_{\sigma \in \frac{1}{3} \mathbb{Z}^{2} / \mathbb{Z}^{2}}
$$

Adopting the notation in $[44, \S 2]$, for any $(j, k) \in\{0,1,2\}^{2}$, we abbreviate

$$
u_{j k}=\Theta_{3}\left[\left(\frac{j}{3}, \frac{k}{3}\right)\right](\tau, 0) \quad \text { and } \quad x_{j k}=\Theta_{3}\left[\left(\frac{j}{3}, \frac{k}{3}\right)\right](\tau, z) .
$$

The nine theta constants $u_{j k}$ satisfy $u_{01}=u_{02}, u_{10}=u_{20}, u_{11}=u_{22}$, and $u_{12}=u_{21}$. For that reason, we need only five theta constants $u_{00}, u_{01}, u_{10}, u_{11}, u_{12}$, which we take as homogeneous coordinates on $\mathbb{P}^{4}$. These five coordinates satisfy one homogeneous equation: 
Lemma 3.3. The closure of the image of the map $\mathfrak{H}_{2} \rightarrow \mathbb{P}^{4}$ given by the five theta constants is an irreducible hypersurface $\mathcal{H}$ of degree 10 . Its defining polynomial is the determinant of

$$
U=\left[\begin{array}{ccccc}
u_{00}^{2} & u_{01}^{2} & u_{10}^{2} & u_{11}^{2} & u_{12}^{2} \\
u_{01}^{2} & u_{00} u_{01} & u_{11} u_{12} & u_{10} u_{12} & u_{10} u_{11} \\
u_{10}^{2} & u_{11} u_{12} & u_{00} u_{10} & u_{01} u_{12} & u_{01} u_{11} \\
u_{11}^{2} & u_{10} u_{12} & u_{01} u_{12} & u_{00} u_{11} & u_{01} u_{10} \\
u_{12}^{2} & u_{10} u_{11} & u_{01} u_{11} & u_{01} u_{10} & u_{00} u_{12}
\end{array}\right]
$$

Proof. This determinant appears in [21, (10)], [27, p. 252], and [40, §2.2].

At this point, we have left the complex analytic world and we are back over a more general field $K$. The natural map $\mathcal{H} \rightarrow \mathcal{B}$ is 10 -to- 1 and it is given explicitly by $4 \times 4$-minors of $U$.

Corollary 3.4. Over the Hessian $\mathcal{H}$ of the Burkhardt quartic, the Coble cubic is written as

$$
C=\operatorname{det}\left[\begin{array}{ccccc}
f(\mathbf{x}) & g_{01}(\mathbf{x}) & g_{10}(\mathbf{x}) & g_{11}(\mathbf{x}) & g_{12}(\mathbf{x}) \\
u_{01}^{2} & u_{00} u_{01} & u_{11} u_{12} & u_{10} u_{12} & u_{10} u_{11} \\
u_{10}^{2} & u_{11} u_{12} & u_{00} u_{10} & u_{01} u_{12} & u_{01} u_{11} \\
u_{11}^{2} & u_{10} u_{12} & u_{01} u_{12} & u_{00} u_{11} & u_{01} u_{10} \\
u_{12}^{2} & u_{10} u_{11} & u_{01} u_{11} & u_{01} u_{10} & u_{00} u_{12}
\end{array}\right]
$$

For $K=\mathbb{C}$, this expresses $r, s_{01}, s_{10}, s_{11}, s_{12}$ as modular forms in terms of theta constants.

We note that the 10 -to- 1 map $\mathcal{H} \rightarrow \mathcal{B}$ is analogous to the 64 -to-1 map in [44, (7.1)] from the Satake hypersurface onto the Göpel variety. The formula for the Coble cubic in Corollary 3.4 is analogous to the expression for the Coble quartic in [44, Theorem 7.1].

In this section we have now introduced four variants of a universal abelian surface. Each of these is a five-dimensional projective variety. Our universal abelian surfaces reside

(a) in $\mathbb{P}^{3} \times \mathbb{P}^{8}$ with coordinates $(\mathbf{c}, \mathbf{x})$,

(b) in $\mathcal{B} \times \mathbb{P}^{8} \subset \mathbb{P}^{4} \times \mathbb{P}^{8}$ with coordinates $\left(\left(r: s_{i j}\right), \mathbf{x}\right)$,

(c) in $\mathcal{B} \times \mathbb{P}^{8} \subset \mathbb{P}^{39} \times \mathbb{P}^{8}$ with coordinates $(\mathbf{m}, \mathbf{x})$,

(d) in $\mathcal{H} \times \mathbb{P}^{8} \subset \mathbb{P}^{4} \times \mathbb{P}^{8}$ with coordinates $(\mathbf{u}, \mathbf{x})$.

A natural commutative algebra problem is to identify explicit minimal generators for the bihomogeneous prime ideals of each of these universal abelian surfaces.

For instance, consider case $(d)$. The ideal contains the polynomial $\operatorname{det}(U)$ of bidegree $(10,0)$ and eight polynomials of bidegree $(8,2)$, namely the partial derivatives of $C$ with respect to the $x_{i j}$. However, these nine do not suffice. For instance, we have ten linearly independent ideal generators of bidegree $(3,3)$, namely the $2 \times 2$-minors of the $2 \times 5$-matrix

$$
\left[\begin{array}{lllll}
f(\mathbf{x}) & g_{01}(\mathbf{x}) & g_{10}(\mathbf{x}) & g_{11}(\mathbf{x}) & g_{12}(\mathbf{x}) \\
f(\mathbf{u}) & g_{01}(\mathbf{u}) & g_{10}(\mathbf{u}) & g_{11}(\mathbf{u}) & g_{12}(\mathbf{u})
\end{array}\right]
$$

These equations have been verified numerically using Sage [49]. For a fixed general point $\mathbf{u} \in \mathcal{S}$, these $2 \times 2$-minors give Gunji's three cubics that were mentioned in Theorem 3.2.

For the case (a) here is a concrete conjecture concerning the desired prime ideal.

Conjecture 3.5. The prime ideal of the universal abelian surface in $\mathbb{P}^{3} \times \mathbb{P}^{8}$ is minimally generated by 93 polynomials, namely 9 polynomials of bidegree $(4,2)$ and 84 of bidegree $(3,3)$. 
The 84 polynomials of bidegree $(3,3)$ are obtained as the $6 \times 6$-subpfaffians of the matrix

$$
\left[\begin{array}{ccccccccc}
0 & -c_{0} x_{02} & c_{0} x_{01} & -c_{1} x_{20} & -c_{2} x_{22} & -c_{3} x_{21} & c_{1} x_{10} & c_{3} x_{12} & c_{2} x_{11} \\
c_{0} x_{02} & 0 & -c_{0} x_{00} & -c_{3} x_{22} & -c_{1} x_{21} & -c_{2} x_{20} & c_{2} x_{12} & c_{1} x_{11} & c_{3} x_{10} \\
-c_{0} x_{01} & c_{0} x_{00} & 0 & -c_{2} x_{21} & -c_{3} x_{20} & -c_{1} x_{22} & c_{3} x_{11} & c_{2} x_{10} & c_{1} x_{12} \\
c_{1} x_{20} & c_{3} x_{22} & c_{2} x_{21} & 0 & -c_{0} x_{12} & c_{0} x_{11} & -c_{1} x_{00} & -c_{2} x_{02} & -c_{3} x_{01} \\
c_{2} x_{22} & c_{1} x_{21} & c_{3} x_{20} & c_{0} x_{12} & 0 & -c_{0} x_{10} & -c_{3} x_{02} & -c_{1} x_{01} & -c_{2} x_{00} \\
c_{3} x_{21} & c_{2} x_{20} & c_{1} x_{22} & -c_{0} x_{11} & c_{0} x_{10} & 0 & -c_{2} x_{01} & -c_{3} x_{00} & -c_{1} x_{02} \\
-c_{1} x_{10} & -c_{2} x_{12} & -c_{3} x_{11} & c_{1} x_{00} & c_{3} x_{02} & c_{2} x_{01} & 0 & -c_{0} x_{22} & c_{0} x_{21} \\
-c_{3} x_{12} & -c_{1} x_{11} & -c_{2} x_{10} & c_{2} x_{02} & c_{1} x_{01} & c_{3} x_{00} & c_{0} x_{22} & 0 & -c_{0} x_{20} \\
-c_{2} x_{11} & -c_{3} x_{10} & -c_{1} x_{12} & c_{3} x_{01} & c_{2} x_{00} & c_{1} x_{02} & -c_{0} x_{21} & c_{0} x_{20} & 0
\end{array}\right] .
$$

This skew-symmetric $9 \times 9$-matrix was derived by Gruson and Sam [29, §3.2], building on the construction in [30], and it is analogous to the elliptic normal curve in (1.1). The nine principal $8 \times 8$-subpfaffians of (3.9) are $x_{00} C, x_{01} C, \ldots, x_{22} C$, where $C$ is the Coble quartic, now regarded as a polynomial in $(\mathbf{c}, \mathbf{x})$ of bidegree $(4,3)$. Conjecture 3.5 is analogous to [44, Conjecture 8.1]. The nine polynomials of bidegree $(4,2)$ are $\partial C / \partial x_{00}, \partial C / \partial x_{01}, \ldots, \partial C / \partial x_{22}$.

In the remainder of this section we recall the symmetry groups that act on our varieties. First there is the complex reflection group denoted by $\mathrm{G}_{32}$ in the classification of Shephard and Todd [47]. The group $\mathrm{G}_{32}$ is a subgroup of order 155520 in $\mathrm{GL}_{4}(K)$. Precisely 80 of its elements are complex reflections of order 3. As a linear transformation on $K^{4}$, each such complex reflection has a triple eigenvalue 1 and a single eigenvalue $\omega^{ \pm 1}=\frac{1}{2}(-1 \pm \sqrt{-3})$.

The center of $\mathrm{G}_{32}$ is isomorphic to the cyclic group $\mathbb{Z} / 6$. In our coordinates $c_{0}, c_{1}, c_{2}, c_{3}$, the elements of the center are scalar multiplications by 6 th roots of unity. Therefore, this gives an action by $\mathrm{G}_{32} /(\mathbb{Z} / 6)$ on the hyperplane arrangement $\mathrm{G}_{32}$ in $\mathbb{P}^{3}$. In fact, we have

$$
\frac{\mathrm{G}_{32}}{\mathbb{Z} / 6} \simeq \operatorname{PSp}_{4}\left(\mathbb{F}_{3}\right)
$$

The linear map $\mathbb{P}^{3} \hookrightarrow \mathbb{P}^{39}, c \mapsto u$, respects the isomorphism (3.10). The group acts on the $c$-coordinates by the reflections on $K^{4}$, and it permutes the coordinates $u_{i j k \ell}$ via its action on the lines through the origin in $\mathbb{F}_{3}^{4}$. Of course, the group $\operatorname{PSp}_{4}\left(\mathbb{F}_{3}\right)$ also permutes the 40 isotropic planes in $\mathbb{P}^{39}$, and this action is compatible with our monomial map $\mathbb{P}^{39} \rightarrow-\rightarrow \mathbb{P}^{39}$.

\section{Tropicalizing the Burkhardt Quartic}

Our goal is to understand the relationship between classical and tropical moduli spaces for curves of genus two. To this end, in this section, we study the tropicalization of the Burkhardt quartic $\mathcal{B}$. This is a 3 -dimensional fan $\operatorname{trop}(\mathcal{B})$ in the tropical projective torus $\mathbb{T P}^{39}$. We shall see that the tropical compactification of $\mathcal{B}^{\circ}$ equals the Igusa compactification of $\mathcal{A}_{3}(2)$.

The variety $\mathcal{B}$ is the closure of the image of the composition $\mathbb{P}^{3} \hookrightarrow \mathbb{P}^{39} \rightarrow \mathbb{P}^{39}$ of the linear map given by the arrangement $\mathrm{G}_{32}$ and the monomial map given by the $40 \times 40$ matrix $A$ that records incidences of isotropic planes and lines in $\mathbb{F}_{3}^{4}$. To be precise, recall that the source $\mathbb{P}^{39}$ has coordinates $e_{\ell}$ indexed by lines $\ell \subset \mathbb{F}_{3}^{4}$, the target $\mathbb{P}^{39}$ has coordinates $e_{W}$ 
indexed by isotropic planes $W \subset \mathbb{F}_{3}^{4}$, and the linear map $A$ is defined by $A\left(e_{\ell}\right)=\sum_{W \supset \ell} e_{W}$. This implies the representation

$$
\operatorname{trop}(\mathcal{B})=A \cdot \operatorname{Berg}\left(\mathrm{G}_{32}\right) \quad \subset \quad \mathbb{T} \mathbb{P}^{39}
$$

of our tropical threefold as the image under $A$ of the Bergman fan of the matroid of $\mathrm{G}_{32}$. By this we mean the unique coarsest fan structure on the tropical linear space given by the rank 4 matroid on the 40 hyperplanes of $\mathrm{G}_{32}$. This Bergman fan is simplicial, as suggested by the general theory of [5]. We computed its cones using the software TropLi due to Rincón [45].

Lemma 4.1. The Bergman complex of the rank 4 matroid of the complex root system $\mathrm{G}_{32}$ has 170 vertices, 1800 edges and 3360 triangles, so its Euler characteristic equals 1729. The rays and cones of the corresponding Bergman fan $\operatorname{Berg}\left(\mathrm{G}_{32}\right) \subset \mathbb{T P}^{39}$ are described below.

The Euler characteristic is the Möbius number of the matroid, which can also be computed as the product of the exponents $n_{i}$ in [43, Table 2] of the complex reflection group $\mathrm{G}_{32}$ :

$$
1 \cdot 7 \cdot 13 \cdot 19=1729=3360-1800+170-1 \text {. }
$$

See $[44,(9.2)]$ for the corresponding formula for the Weyl group of $\mathrm{E}_{7}$ (and genus 3 curves).

We now discuss the combinatorics of $\operatorname{Berg}\left(G_{32}\right)$. The space $\mathbb{P P}^{39}=\mathbb{R}^{40} / \mathbb{R}(1,1, \ldots, 1)$ is spanned by unit vectors $e_{0001}, e_{0010}, \ldots, e_{1222}$ that are labeled by the 40 lines in $\mathbb{F}_{3}^{4}$ as before. The 170 rays of the Bergman fan correspond to the connected flats of the matroid of $\mathrm{G}_{32}$, and these come in three symmetry classes, according to the rank of the connected flat:

(a) 40 Bergman rays of rank 1 . These are spanned by the unit vectors $e_{0001}, e_{0010}, \ldots, e_{1222}$.

(b) 90 Bergman rays of rank 2 , such as $e_{0001}+e_{0100}+e_{0101}+e_{0102}$, which represents $\left\{c_{1}, c_{1}+c_{2}+c_{3}, c_{1}+\omega c_{2}+\omega c_{3}, c_{1}+\omega^{2} c_{2}+\omega^{2} c_{3}\right\}$. These are the non-isotropic planes in $\mathbb{F}_{3}^{4}$.

(ä) 40 Bergman rays of rank 3, such as

$e_{0001}+e_{0010}+e_{0011}+e_{1100}+e_{1101}+e_{1102}+e_{1110}+e_{1111}+e_{1112}+e_{1120}+e_{1121}+e_{1122}$.

These correspond to the Hesse pencils in $\mathrm{G}_{32}$, and to the hyperplanes in $\mathbb{F}_{3}^{4}$. Note that the 12 indices above are perpendicular to $(0,0,1,2)$ in the symplectic inner product.

The 3360 triangles of the Bergman complex of $\mathrm{G}_{32}$ also come in three symmetry classes:

(aaä) Two orthogonal lines (a) together with a hyperplane (ä) that contains them both. This gives 480 triangles because each hyperplane contains 12 orthogonal pairs.

(abä) A flag consisting of a line (a) contained in a non-isotropic plane (b) contained in a hyperplane (ä). There are 1440 such triangles since each of the 90 planes has 4.4 choices.

(aab) Two orthogonal lines (a) together with a non-isotropic plane (b). The plane contains one of the lines and is orthogonal to the other one. The count is also 1440. 
The 1800 edges of the Bergman complex come in five symmetry classes: there are 240 edges (aa) given by pairs of orthogonal lines, 360 edges (ab) given by lines in non-isotropic planes, 480 edges (ä̈) given by lines in hyperplanes, 360 edges (bä) given by non-isotropic planes in hyperplanes, and 360 edges $\left(\mathrm{ab}^{\perp}\right)$ obtained by dualizing the previous pairs (bä).

Our calculations establish the following statement:

Proposition 4.2. The Bergman complex coincides with the nested set complex for the matroid of $\mathrm{G}_{32}$. In particular, the tropical compactification of the complement of the hyperplane arrangement $\mathrm{G}_{32}$ coincides with the wonderful compactification of de Concini-Procesi [18].

See [24] for the relation between tropical compactifications and wonderful compactifications. We expect that Proposition 4.2 is true for any finite complex reflection group, but we have not made any attempts to prove this.

The wonderful compactification is obtained by blowing up the irreducible flats of lowest dimension, then blowing up the strict transforms of the irreducible flats of next lowest dimension, etc. In our case, the smallest irreducible flats are 40 points, corresponding to the Bergman rays (a) and to Family 6 in [29, Table 1]. This first blow-up $\widehat{\mathbb{P}^{3}}$ is the closure of the graph of the map $\mathbb{P}^{3} \rightarrow-\mathcal{B}$, by [29, Proposition 3.25]. The next smallest irreducible flats are the strict transforms of $90 \mathbb{P}^{1}$ 's, corresponding to the Bergman rays (b) and to Family 4 in [29, Table 1]. After that, the only remaining irreducible flats are 40 hyperplanes, corresponding to the Bergman rays (ä) and to Family 2 in [29, Table 1]. Hence the wonderful compactification $\widetilde{\mathbb{P}^{3}}$ is obtained by blowing up these $90 \mathbb{P}^{1}$ 's in $\widehat{\mathbb{P}^{3}}$. The 90 exceptional divisors of $\widetilde{\mathbb{P}^{3}} \rightarrow \widehat{\mathbb{P}^{3}}$ get contracted to the 45 nodes of $\mathcal{B}$, so we can lift the map $\widetilde{\mathbb{P}^{3}} \rightarrow \mathcal{B}$ to a map $\widetilde{\mathbb{P}^{3}} \rightarrow \widetilde{\mathcal{B}}$, where $\widetilde{\mathcal{B}}$ denotes the blow-up of the Burkhardt quartic at its 45 singular points.

The hyperplane arrangement complement $\mathbb{P}^{3} \cap \mathbb{G}_{m}^{39}$ is naturally identified with the moduli space $\mathcal{M}_{2}(3)^{-}$of smooth genus 2 curves with level 3 structure and the choice of a Weierstrass point (or equivalently, the choice of an odd theta characteristic). See [9] for more about $\mathcal{M}_{2}(3)^{-}$. Hence we have the following commutative diagram

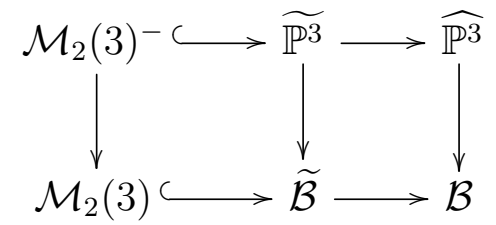

where the vertical maps are generically finite of degree 6, the right horizontal maps are blow-ups, and the moduli spaces $\mathcal{M}_{2}(3)^{-}$and $\mathcal{M}_{2}(3)$ are realized as very affine varieties.

We now compute the tropical Burkhardt quartic (4.1), by applying the linear map $A$ to the Bergman fan of $\mathrm{G}_{32}$. Note that the image lands in the tropicalization $\operatorname{trop}(\mathcal{T})$ of the toric variety $\mathcal{T} \subset \mathbb{P}^{39}$. We regard $\operatorname{trop}(\mathcal{T})$ as a 24 -dimensional linear subspace of $\mathbb{T} \mathbb{P}^{39}$.

Theorem 4.3. The tropical Burkhardt quartic $\operatorname{trop}(\mathcal{B})$ is the fan over a 2-dimensional simplicial complex with 85 vertices, 600 edges and 880 triangles. A census appears in Table 1.

Proof. Given the $\mathrm{G}_{32}$-symmetry, the following properties of the map $A$ can be verified on representatives of $\mathrm{G}_{32}$-orbits. The linear map $A: \mathbb{T P}^{39} \rightarrow \mathbb{T P}^{39}$ has the property that the image of each vector (ä) equals twice that of the corresponding unit vector (a). For instance,

$$
A\left(e_{0001}+e_{0010}+e_{0011}+e_{1100}+e_{1101}+e_{1102}+e_{1110}+e_{1111}+e_{1112}+e_{1120}+e_{1121}+e_{1122}\right)=2 A e_{0012} .
$$


Likewise, the 90 vectors (b) come in natural pairs of non-isotropic planes that are orthogonal complements. The corresponding vectors have the same image under $A$. For instance,

$$
A\left(e_{0001}+e_{0100}+e_{0101}+e_{0102}\right)=A\left(e_{0010}+e_{1000}+e_{1010}+e_{1020}\right) .
$$

We refer to such a pair of orthogonal non-isotropic planes as a plane pair. This explains the 85 rays of $\operatorname{trop}(\mathcal{B})$, namely, they are the 40 lines $a$ and the 45 plane pairs $\left\{b, b^{\perp}\right\}$ in $\mathbb{F}_{3}^{4}$.

The image of each cone of $\operatorname{Berg}\left(\mathrm{G}_{32}\right)$ under $A$ is a simplicial cone of the same dimension. There are no non-trivial intersections of image cones. The map $\operatorname{Berg}\left(\mathrm{G}_{32}\right) \rightarrow \operatorname{trop}(\mathcal{B})$ is a proper covering of fans. The 2 -to- 1 covering of the rays induces a 3 -to- 1 or 4 -to- 1 covering on each higher-dimensional cone. The precise combinatorics is summarized in Table 1.

\begin{tabular}{|c|c|c|c|c|}
\hline Dimension & Orbits in $\operatorname{Berg}\left(\mathrm{G}_{32}\right)$ & The map $A$ & Orbit size in $\operatorname{trop}(\mathcal{B})$ & Cone type \\
\hline \multirow{3}{*}{1} & $40(\mathrm{a})$ & \multirow{2}{*}{2 to 1} & \multirow{2}{*}{40} & \multirow{2}{*}{ (a) } \\
\hline & $40(\ddot{a})$ & & & \\
\hline & $90(\mathrm{~b})$ & 2 to 1 & 45 & (b) \\
\hline \multirow{5}{*}{2} & $240(\mathrm{aa})$ & \multirow{2}{*}{3 to 1} & \multirow{2}{*}{240} & \multirow{2}{*}{ (aa) } \\
\hline & 480 (aӓ) & & & \\
\hline & $360(a b)$ & \multirow{3}{*}{3 to 1} & \multirow{3}{*}{360} & \multirow{3}{*}{$(a b)$} \\
\hline & 360 (bä) & & & \\
\hline & $360\left(a b^{\perp}\right)$ & & & \\
\hline \multirow{3}{*}{3} & $1440(\mathrm{aab})$ & \multirow{2}{*}{4 to 1} & \multirow{2}{*}{720} & \multirow{2}{*}{$(\mathrm{aab})$} \\
\hline & 1440 (abä) & & & \\
\hline & 480 (aаä) & 3 to 1 & 160 & (aaa) \\
\hline
\end{tabular}

Table 1: Orbits of cones in the tropical Burkhardt quartic

The types of the cones are named by replacing $\ddot{a}$ with $a$, and $b^{\perp}$ with $b$. In total, there are 40 vertices of type (a) and 45 vertices of type (b). There are 240 edges of type (aa), corresponding to pairs of lines $a \perp a^{\prime}$, and 360 edges of type (ab), corresponding to inclusions $a \subset b$. Finally, there are 160 triangles of type (aaa) and 720 triangles of type (aab).

Remark 4.4. There is a bijection between the 45 rays of type (b) in $\operatorname{trop}(\mathcal{B})$ and the 45 singular points in $\mathcal{B}$. Namely, each vector of type (b) can be written such that 16 of its coordinates are 1 and the other coordinates are 0 . These 16 coordinates are exactly the 16 zero coordinates in the corresponding singular point. Note that the zero coordinates of the particular singular point in (3.4) form precisely the support of the vector (4.3). The number 16 arises because each of the 45 plane pairs $\left\{b, b^{\perp}\right\}$ determines 16 of the 40 isotropic planes: take any vector in $b$ and any vector in $b^{\perp}$, and these two will span an isotropic plane.

We next consider the tropical compactification $\overline{\mathcal{B}}$ of the open Burkhardt quartic $\mathcal{B}^{\circ}=$ $\mathcal{M}_{2}(3)$. By definition, $\overline{\mathcal{B}}$ is the closure of $\mathcal{B}^{\circ} \subset \mathbb{G}_{m}^{24}$ inside of the toric variety defined by the fan $\operatorname{trop}(\mathcal{B})$. This toric variety is smooth because the rays of the two types of maximal cones (aaa) and (aab) can be completed to a basis of the lattice $\mathbb{Z}^{24}$ spanned by all 85 rays.

Proposition 4.5. The tropical compactification $\overline{\mathcal{B}}$ is schön in the sense of Tevelev [50]. The boundary $\overline{\mathcal{B}} \backslash \mathcal{B}^{\circ}$ is a normal crossing divisor consisting of 85 irreducible smooth surfaces. 
Proof. Since our fan on $\operatorname{trop}(\mathcal{B})$ defines a smooth toric variety, it suffices to show that all initial varieties $V\left(\operatorname{in}_{v}\left(I_{\mathcal{B}}\right)\right)$ are smooth and connected in the torus $\mathbb{G}_{m}^{39}$ [32, Lemma 2.7]. There are six symmetry classes of initial ideals $\operatorname{in}_{v}\left(I_{\mathcal{B}}\right)$. Each of them is generated by linear binomials and trinomials together with one non-linear polynomial $f$, obtained from the quartic by possibly removing monomial factors. We present representatives for the six classes. The plane pair $\left\{b, b^{\perp}\right\}$ appearing in three of the cases is precisely the one displayed in (4.3).

(a) For the vertex given by the line $(0,0,0,1)$ we take the weight vector $v=A e_{0001}=$ $e_{0}+e_{1}+e_{2}+e_{3}$. Then $f=m_{0} m_{4}^{3}-3 m_{0} m_{4} m_{7} m_{10}+m_{0} m_{7}^{3}+m_{0} m_{10}^{3}-3 \sqrt{3} m_{1} m_{4} m_{7} m_{10}$. This bihomogeneous polynomial defines a smooth surface in a quotient torus $\mathbb{G}_{m}^{3}$.

(b) For the vertex $\left\{b, b^{\perp}\right\}$ we take the vector (4.3). This is the incidence vector of the zero coordinates in (3.4), namely $v=e_{0}+e_{1}+e_{2}+e_{3}+e_{4}+e_{5}+e_{6}+e_{13}+e_{14}+e_{15}+e_{16}+$ $e_{17}+e_{18}+e_{19}+e_{20}+e_{21}$. The resulting non-linear polynomial is $f=-m_{0} m_{13}+m_{1} m_{4}$.

(aa) For the edge given by the orthogonal lines $(0,0,0,1)$ and $(0,0,1,0)$ in $\mathbb{F}_{3}^{4}$, we take $v=2 e_{0}+e_{1}+e_{2}+e_{3}+e_{4}+e_{5}+e_{6}$, and we get $f=m_{0} m_{7}^{3}+m_{0} m_{10}^{3}-3 \sqrt{3} m_{1} m_{4} m_{7} m_{10}$.

(ab) For the edge given by $(0,0,0,1)$ and $\left\{b, b^{\perp}\right\}$, we take $v=2 e_{0}+2 e_{1}+2 e_{2}+2 e_{3}+e_{4}+$ $e_{5}+e_{6}+e_{13}+e_{14}+e_{15}+e_{16}+e_{17}+e_{18}+e_{19}+e_{20}+e_{21}$, and we get $f=-m_{0} m_{13}+m_{1} m_{4}$.

(aaa) For the triangle given by $(0,0,0,1),(0,0,1,0)$ and $(0,0,1,1)$, we take $v=A\left(e_{0001}+e_{0010}\right.$ $\left.+e_{0011}\right)=3 e_{0}+e_{1}+e_{2}+e_{3}+e_{4}+e_{5}+e_{6}+e_{7}+e_{8}+e_{9}$, and we get $f=m_{0} m_{10}^{2}-3 \sqrt{3} m_{1} m_{4} m_{7}$.

(aab) For the triangle given by $(0,0,0,1),(0,0,1,0)$ and $\left\{b, b^{\perp}\right\}$, we take $v=3 e_{0}+2 e_{1}+2 e_{2}+$ $2 e_{3}+2 e_{4}+2 e_{5}+2 e_{6}+e_{13}+e_{14}+e_{15}+e_{16}+e_{17}+e_{18}+e_{19}+e_{20}+e_{21}$. Here, $f=-m_{0} m_{13}+m_{1} m_{4}$.

Note that the polynomials $f$ are the same in the cases (b), (ab) and (aab), but the varieties $V\left(\operatorname{in}_{v}\left(I_{\mathcal{B}}\right)\right)$ are different because of the 35 linear relations. In cases (b) and (ab) we have both linear trinomials and linear binomials, while in case (aab) they are all binomials. In all six cases the hypersurface $\{f=0\}$ has no singular points with all coordinates nonzero.

Our final goal in this section is to equate the tropical compactification $\overline{\mathcal{B}}$ with the blown up Burkhardt quartic $\widetilde{\mathcal{B}}$. By [36, Theorem 5.7.2], we can identify $\widetilde{\mathcal{B}}$ with the Igusa desingularization of the Baily-Borel-Satake compactification $\mathcal{A}_{2}(3)^{\text {Sat }}$ of $\mathcal{A}_{2}(3)$. The latter can be constructed as follows. Let $\widehat{\mathcal{B}}$ be the projective dual variety to $\mathcal{B} \subset \mathbb{P}^{4}$. The canonical birational map $\mathcal{B} \rightarrow \widehat{\mathcal{B}}$ is defined outside of the 45 nodes. Since $\mathcal{B}$ is a normal variety, this map factors through the normalization of $\widehat{\mathcal{B}}$, which can be identified with $\mathcal{A}_{2}(3)^{\text {Sat }}$ by $[26$, $\S 4]$. The closure of the graph of the birational map $\mathcal{B} \rightarrow-\mathcal{A}_{2}(3)^{\text {Sat }}$ is the blow-up of $\mathcal{B}$ at its indeterminacy locus, i.e., the 45 nodes. Using [52, Theorem 3.1], we may identify this with $\widetilde{\mathcal{B}}$. By symmetry, we could also view this as the closure of the image of the inverse birational map. This realizes $\widetilde{\mathcal{B}}$ as a blow-up of $\mathcal{A}_{2}(3)^{\text {Sat }}$, and in particular, the map blows up the Satake boundary $\mathcal{A}_{2}(3)^{\text {Sat }} \backslash \mathcal{A}_{2}(3)$ which has 40 components all isomorphic to $\mathcal{A}_{1}(3)^{\text {Sat }} \cong \mathbb{P}^{1}$.

Lemma 4.6. The moduli space $\mathcal{A}_{2}(3)$ coincides with the partial compactification of $\mathcal{M}_{2}(3)$ given by the 1-dimensional subfan of $\operatorname{trop}(\mathcal{B})$ that consists of the 45 rays of type $(b)$. 
Proof. Let $M$ be the partial compactification in question. Let $\widetilde{\mathbb{P}^{3}}$ be the wonderful compactification for $\mathrm{G}_{32}$ as described above. The preimage of the 45 rays of type (b) in $\operatorname{Berg}\left(\mathrm{G}_{32}\right)$ consists of 90 rays, and the resulting partial tropical compactification $P$ of $\mathbb{P}^{3} \backslash$ (40 hyperplanes) is the complement of the strict transforms of the reflection hyperplanes in $\widetilde{\mathbb{P}^{3}}$. In the map $P \rightarrow \mathcal{B}$, the 90 divisors are contracted to the 45 singular points (2 divisors to each point). We have a map $P \rightarrow M$ which maps the 90 divisors of $P$ to the 45 divisors of $M$, and hence the birational map $M-\rightarrow \mathcal{B}$ (given by the identity map on $\mathcal{M}_{2}(3)$ ) extends to a regular map $M \rightarrow \mathcal{B}$ which contracts the 45 divisors to the 45 singular points.

By the universal property of blow-ups, there exists a map $M \rightarrow \widetilde{\mathcal{B}}$ which takes each of the 45 divisors to one of the 45 exceptional divisors of the blow-up $\widetilde{\mathcal{B}} \rightarrow \mathcal{B}$. From our previous discussion, the image of the map $P \rightarrow \widetilde{\mathcal{B}}$ equals $\mathcal{A}_{2}(3)$. Since this map factors through $M$, the image of the map $M \rightarrow \widetilde{\mathcal{B}}$ is also $\mathcal{A}_{2}(3)$. This map has finite fibers: this just needs to be checked on the 45 divisors and we can reduce to considering the map $P \rightarrow \widetilde{\mathcal{B}}$; in the map $\widehat{\mathbb{P}^{3}} \rightarrow \widetilde{\mathcal{B}}$, the inverse image of an exceptional divisor is 2 disjoint copies of $\mathbb{P}^{1} \times \mathbb{P}^{1}$ and any surjective endomorphism of $\mathbb{P}^{1} \times \mathbb{P}^{1}$ has finite fibers. The map is birational and $\mathcal{A}_{2}(3)$ is smooth, so, by Zariski's Main Theorem [41, §III.9], the map is an isomorphism.

Theorem 4.7. The intrinsic torus of $\mathcal{M}_{2}(3)=\mathcal{B}^{\circ}$ is the dense torus $\mathbb{G}_{m}^{24}$ of the toric variety $\mathcal{T}$ described in Proposition 3.1. The tropical compactification of $\mathcal{M}_{2}(3)$ provided by $\operatorname{trop}(\mathcal{B})$ is the Igusa desingularization $\widetilde{\mathcal{B}}$ of the Baily-Borel-Satake compactification $\mathcal{A}_{2}(3)^{\text {Sat }}$ of $\mathcal{A}_{2}(3)$.

Proof. The first statement follows from Lemma 2.6 and Proposition 3.1(a).

By Lemma $4.6, \overline{\mathcal{B}}$ is a compactification of $\mathcal{A}_{2}(3)$. The boundary of the compactification $\mathcal{M}_{2}(3) \subset \overline{\mathcal{B}}$ is a normal crossings divisor (Proposition 4.5), so the same is true for the boundary of $\mathcal{A}_{2}(3) \subset \overline{\mathcal{B}}$, and hence it is toroidal. So there exists a map $f: \overline{\mathcal{B}} \rightarrow \mathcal{A}_{2}(3)^{\text {Sat }}$ that is the identity on $\mathcal{A}_{2}(3)$ [10, Proposition III.15.4(3)]. This map is unique and surjective.

From what we said above, the Satake boundary $\mathcal{A}_{2}(3)^{\text {Sat }} \backslash \mathcal{A}_{2}(3)$ has 40 components all isomorphic to $\mathbb{P}^{1}$. Also, $\overline{\mathcal{B}} \backslash \mathcal{A}_{2}(3)$ consists of 40 divisors. Hence the map $f$ contracts the 40 divisors to these $\mathbb{P}^{1}$ 's. By the universal property of blow-ups, there is a unique map $\tilde{f}: \overline{\mathcal{B}} \rightarrow \widetilde{\mathcal{B}}$ that commutes with the blow-up map. Then $\tilde{f}$ is birational and surjective. We know this map is an isomorphism on $\mathcal{A}_{2}(3)$ and the complement of this open subset in both domain and target are a union of $\mathbb{P}^{2}$ 's. Any surjective endomorphism of $\mathbb{P}^{2}$ has finite fibers, and hence $\tilde{f}$ is an isomorphism by Zariski's Main Theorem [41, §III.9] since $\widetilde{\mathcal{B}}$ is smooth.

\section{Moduli of Genus Two Curves}

The moduli space $\mathcal{M}_{g, n}^{\mathrm{tr}}$ of tropical curves of genus $g$ with $n$ marked points is a stacky fan. This was shown by Brannetti, Melo and Viviani [11] and Chan [15]. This space was studied by many authors. See $[12,13]$ for some results. Here, a tropical curve is a triple $(\Gamma, w, \ell)$, where $\Gamma=(V, E)$ is a connected graph, $w$ is a weight function $V \rightarrow \mathbb{Z}_{\geq 0}$, and $\ell$ is a length function $E \rightarrow \mathbb{R}_{\geq 0}$. The genus of a tropical curve is the sum of weights of all vertices plus the genus of the graph $\Gamma$. In addition to identifications induced by graph automorphisms, two tropical curves are isomorphic if one can be obtained from another by a sequence of the following operations and their inverses: 
- Removing a leaf of weight 0 , together with the only edge connected to it.

- Removing a vertex of degree 2 of weight 0 , and replacing the two edges connected to it with an edge whose length is the sum of the two old edges.

- Removing an edge of length 0 , and combining the two vertices connected by that edge. The weight of the new vertex is the sum of the two old vertices.

In this way, every tropical curve of genus $\geq 2$ is uniquely represented by a minimal skeleton, i.e., a tropical curve with no vertices of weight 0 of degree $\leq 2$ or edges of length 0 . The moduli space of tropical curves with a fixed combinatorial type $(\Gamma, w)$ is $\mathbb{R}_{>0}^{|E|} / \operatorname{Aut}(\Gamma)$, where the coordinates of $\mathbb{R}_{>0}^{|E|}$ are the lengths of the edges. The cones for all combinatorial types are glued together to form $\mathcal{M}_{g}^{\text {tr }}$. The boundary of the cone of a combinatorial type $(\Gamma, w)$ corresponds to tropical curves with at least one edge of length 0. Contracting that edge gives a combinatorial type $\left(\Gamma^{\prime}, w^{\prime}\right)$. Then, the cone for $\left(\Gamma^{\prime}, w^{\prime}\right)$ is glued along the boundary of the cone for $(\Gamma, w)$ in the natural way. More generally, a tropical curve with marked points is defined similarly, but allowing rays connecting a vertex with leaves "at infinity".

The following construction maps curves over a valued field to tropical curves. It is fundamental for [3, 7]. Our description follows [54, Lemma - Definition 2.2.4]. Let $R$ be a complete discrete valuation ring with maximal ideal $\mathfrak{m}$. Let $K$ be its field of fractions, $k=R / \mathfrak{m}$ its residue field, and $t \in R$ a uniformizing parameter. Fix a genus $g$ curve $C$ with $n$ marked points over $K$. The curve $C$ is a morphism Spec $K \rightarrow \mathcal{M}_{g, n}$. Since the stack $\overline{\mathcal{M}}_{g, n}$ is proper (i.e., by the stable reduction theorem), there is a finite extension $K^{\prime}$ of $K$ with discrete valuation ring $R^{\prime}$ such that this morphism extends uniquely to a morphism Spec $R^{\prime} \rightarrow \overline{\mathcal{M}}_{g, n}$ (we call this a stable model of $C$ ). Here we renormalize the valuation on $R^{\prime}$ so that its value group is $\mathbb{Z}$. Reducing modulo $\mathfrak{m}^{\prime}$ gives us a point $\operatorname{Spec} k \rightarrow \overline{\mathcal{M}}_{g, n}$. By definition, this is a stable curve $C_{k}$ over $k$. We remark that the stable model may not be unique, but the stable curve is unique. Since such a stable curve has at worst nodal singularities, we can construct a dual graph as follows. For each genus $h$ component of $C_{k}$, we draw a vertex of weight $h$. For each node of $C_{k}$, we draw an edge between the two components that meet there (this might be a loop if the node comes from a self-intersection). If a component has a marked point, then we attach a vertex at infinity to that vertex. The stable condition translates to the fact that the dual graph is a minimal skeleton as above. Finally, each node, when considered as a point in $C_{R^{\prime}}$, is étale locally of the form $x y=t^{\ell}$ for some positive integer $\ell$. We then assign the length $\ell / d$ to the corresponding edge, where $d$ is the degree of the field extension $K \subset K^{\prime}$. In this way, we have defined a function

$$
\mathcal{M}_{g, n}(K) \rightarrow \mathcal{M}_{g, n}^{\text {tr }}
$$

Here is a concrete illustration of this function for $g=0$ and $n=4$.

Example 5.1. Let $K=\mathbb{C}((t))$ and $R=\mathbb{C} \llbracket t \rrbracket$ and consider the four points in $\mathbb{P}_{K}^{1}$ given by $(1: p(t)),(1: q(t)),(1: a),(1: b)$ where $a, b \in \mathbb{C}$ are generic and val $(p(t))$, val $(q(t))>0$. Let $x, y$ be the coordinates on $\mathbb{P}^{1}$. Naively, this gives us four points in $\mathbb{P}_{R}^{1}$, but it is not a stable model since $p(0)=q(0)=0$ and so two points coincide in the special fiber. The fix is to blow-up the arithmetic surface $\mathbb{P}_{R}^{1}$ at the ideal $\langle y-p(t) x, y-q(t) x\rangle$. We embed this blow-up into $\mathbb{P}_{R}^{1} \times{ }_{R} \mathbb{P}_{R}^{1}$, where the latter $\mathbb{P}_{R}^{1}$ has coordinates $w, z$, as the hypersurface given by $w(y-q(t) x)=z(y-p(t) x)$. The special fiber is the nodal curve given by $y(w-z)=0$. We wish to understand the étale local equation for the node cut out by $y=w-z=0$. To 
do this, set $x=z=1$ and consider the defining equation $y(w-1)+p(t)-q(t) w=0$. Now substitute $w^{\prime}=w-1$ and $y^{\prime}=y-q(t)$ to get $y^{\prime} w^{\prime}+(p(t)-q(t))=0$. Hence the dual curve is a line segment of length $\operatorname{val}(p(t)-q(t))$ with both vertices having weight 0 .

Evaluating the map (5.1) in general is a challenging computer algebra problem: how does one compute the metric graph from a smooth curve $C$ that is given by explicit polynomial equations over $K$ ? This section represents a contribution to this problem for curves of genus 2. As a warm-up for our study of genus 2 curves, let us first consider the genus 1 case.

Example 5.2. An elliptic curve $C$ can be defined by giving four points in $\mathbb{P}^{1}$. The curve is the double cover of $\mathbb{P}^{1}$ branched at those four points. This gives us a map $\mathcal{M}_{0,4} \rightarrow \mathcal{M}_{1}$, which is well-defined over our field $K$. The map is given explicitly by the following formula for the j-invariant of $C$ in terms of the cross ratio $\lambda$ of four ramification points (see $[51, \S 3]$ ):

$$
j=256 \frac{\left(\lambda^{2}-\lambda+1\right)^{3}}{\lambda^{2}(\lambda-1)^{2}} .
$$

We now pass to the tropicalization by constructing a commutative square

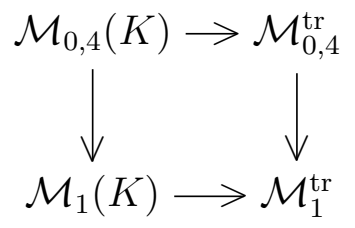

The horizontal maps are instances of (5.1), and the left vertical map is (5.2). Our task is to define the right vertical map. The ingredients are the trees and tropical curves in Table 2 :

\begin{tabular}{|l|l|}
\hline Tropical curve of genus 1 & Tree with 4 leaves \\
\hline
\end{tabular}

Table 2: Trees on four taxa and tropical curves of genus 1

A point in $\mathcal{M}_{0,4}^{\mathrm{tr}}$ can be represented by a phylogenetic tree with taxa $1,2,3,4$. Writing $\nu_{i j}$ for half the distance from leaf $i$ to leaf $j$ in that tree, the unique interior edge has length

$$
\ell=\max \left\{\nu_{12}+\nu_{34}-\nu_{14}-\nu_{23}, \nu_{13}+\nu_{24}-\nu_{12}-\nu_{34}, \nu_{14}+\nu_{23}-\nu_{13}-\nu_{24}\right\} .
$$

Suppose we represent a point in $\mathcal{M}_{0,4}$ by four scalars, $x_{1}, x_{2}, x_{3}, x_{4} \in K$, as in Example 5.1. Then its image in $\mathcal{M}_{0,4}^{\mathrm{tr}}$ is the phylogenetic tree obtained by setting

$$
\nu_{i j}=-\operatorname{val}\left(x_{i}-x_{j}\right) .
$$


The square (5.3) becomes commutative if the right vertical map takes trees with interior edge length $\ell>0$ to the cycle of length $2 \ell$, and it takes the star tree $(\ell=0)$ to the node marked 1 . To see this, we recall that the tropical curve contains a cycle of length $-\operatorname{val}(j)$, where $j$ is the $\mathrm{j}$-invariant. This is a standard fact (see $[7, \S 7]$ ) from the theory of elliptic curves over $K$. Suppose the four given points in $\mathbb{P}^{1}$ are $0,1, \infty, \lambda$, and that $\lambda$ and 0 are neighbors in the tree. This means $\operatorname{val}(\lambda)>0$. As desired, the length of our cycle is

$$
-\operatorname{val}(j)=-3 \operatorname{val}\left(\lambda^{2}-\lambda+1\right)+2 \operatorname{val}(\lambda)+2 \operatorname{val}(\lambda-1)=0+2 \operatorname{val}(\lambda)+0=2 \operatorname{val}(\lambda) .
$$

The other case, when $\lambda$ and 0 are not neighbors in the tree, follows from the fact that the rational function of $\lambda$ in (5.2) is invariant under permuting the four ramification points.

In this example, the one-dimensional fan $\mathcal{M}_{0,4}^{\text {tr }}$ serves as a moduli space for tropical elliptic curves. A variant where the fibers are elliptic normal curves is shown in Figure 2. In both situations, all maximal cones correspond to elliptic curves over $K$ with bad reduction.

Moving on to genus 2 curves, we shall now focus on the tropical spaces $\mathcal{M}_{2}^{\operatorname{tr}}$ and $\mathcal{M}_{0,6}^{\operatorname{tr}}$. There are seven combinatorial types for genus 2 tropical curves. Their poset is shown in $[15$, Figure 4]. The seven types are drawn in the second column of Table 3 . The stacky fan $\mathcal{M}_{2}^{\text {tr }}$ is the cone over the two-dimensional cell complex shown in Figure 5. Note the identifications.

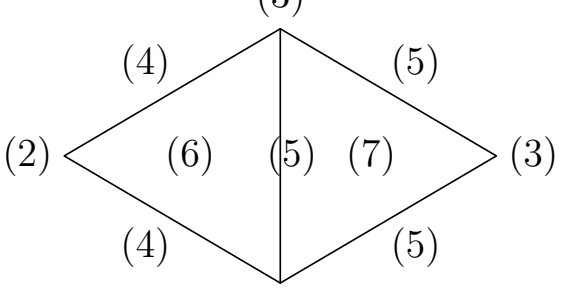

(3)

Figure 5: The moduli space of genus 2 tropical curves

The tropical moduli space $\mathcal{M}_{0,6}^{\mathrm{tr}}$ is the space of phylogenetic trees on six taxa. A concrete model, embedded in $\mathbb{T P}^{14}$, is the 3-dimensional fan $\operatorname{trop}\left(\mathcal{M}_{0,6}\right)$ seen in Theorem 2.4. Combinatorially, it agrees with the tropical Grassmannian $\operatorname{Gr}(2,6)$ as described in [48, Example 4.1], so its cones correspond to trees with six leaves. The fan $\mathcal{M}_{0,6}^{\text {tr }}$ has one zero-dimensional cone of type (1), $25=10+15$ rays of types (2) and (3), $105=60+45$ two-dimensional cones of types (4) and (5), and $105=90+15$ three-dimensional cones of types (6) and (7). The corresponding combinatorial types of trees are depicted in the last column of Table 3.

Table 3 shows that there is a combinatorial correspondence between the types of cones of the tropical Burkhardt quartic $\operatorname{trop}(\mathcal{B})$ in Table 1 and the types of cones in $\mathcal{M}_{2}^{\operatorname{tr}}$ and $\mathcal{M}_{0,6}^{\operatorname{tr}}$. We seek to give a precise explanation of this correspondence in terms of algebraic geometry. At the moment we can carry this out for level 2 but we do not yet have a proof for level 3 .

Theorem 5.3. Let $K$ be a complete nonarchimedean field.

(a) There is a commutative square

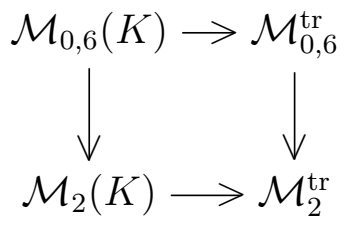




\begin{tabular}{|c|c|c|c|}
\hline Label & Tropical curve of genus 2 & Burkhardt cone & Tree with 6 leaves \\
\hline$(1)$ & 2 . & origin & \\
\hline$(2)$ & $1 \bullet \longrightarrow 1$ & (b) & \\
\hline$(3)$ & & (a) & \\
\hline$(4)$ & & $(a b)$ & \\
\hline$(5)$ & & (aa) & \\
\hline$(6)$ & & $(a a b)$ & \\
\hline$(7)$ & & (aaa) & $\checkmark$ \\
\hline
\end{tabular}

Table 3: Correspondence between tropical curves, cones of $\operatorname{trop}(\mathcal{B})$, and metric trees. 
The left vertical map sends 6 points in $\mathbb{P}^{1}$ to the genus 2 hyperelliptic curve with these ramification points. The horizontal maps send a curve (with or without marked points) to its tropical curve (with or without leaves at infinity). The right vertical map is a morphism of generalized cone complexes relating the second and fourth columns of Table 3 .

(b) The top horizontal map can be described in an alternative way: under the embedding of $\mathcal{M}_{0,6}$ into $\mathbb{P}^{14}$ given by $(2.2)$, take the valuations of the 15 coordinates $m_{0}, m_{1}, \ldots, m_{14}$.

Proof of Theorem 5.3. We start with (a). Let $C$ be a genus 2 curve over $K$ and let $p_{1}, \ldots, p_{6} \in$ $\mathbb{P}_{K}^{1}$ be the branch points of the double cover $C \rightarrow \mathbb{P}^{1}$ induced from the canonical divisor. Let $R^{\prime}$ be a discrete valuation ring over which a stable model of both $C$ and $\left(\mathbb{P}^{1}, p_{1}, \ldots, p_{6}\right)$ can be defined and let $k$ be its residue field. The fact that the combinatorial types of dual graphs for $C$ and the marked curve $\left(\mathbb{P}^{1}, p_{1}, \ldots, p_{6}\right)$ match up as in Table 3 is clear from the proof of [6, Corollary 2.5] which constructs the stable $k$-curve of $C$ from that of $\left(\mathbb{P}^{1}, p_{1}, \ldots, p_{6}\right)$. There is an obvious bijection of edges between the combinatorial types in all cases. We claim that the edge length coming from the étale neighborhood of nodal singularities is halved for curves of type (2) and is doubled for curves of type (3) from Table 3: the description and proof for the other types can be reduced to these two cases.

First consider curves of type (3). Our stable genus 0 curve consists of the union of two $\mathbb{P}^{1}$ 's meeting in a point. One has 4 marked points and the other has 2 marked points. This arises from 6 distinct points in $\mathbb{P}_{R^{\prime}}^{1}$ such that exactly 2 of them coincide after passing to the residue field. To build a stable model (cf. Example 5.1), we blow up the point of intersection in the special fiber of $\mathbb{P}_{R^{\prime}}^{1}$ to get an arithmetic surface $\tilde{P}_{R^{\prime}}$. Let $E$ be the double cover of the first $\mathbb{P}_{k}^{1}$ along the 4 marked points, and let $E^{\prime}$ be a copy of $\mathbb{P}_{k}^{1}$ mapping to the second $\mathbb{P}_{k}^{1}$ so that it is ramified over the 2 marked points. Over the point of intersection, both $E$ and $E^{\prime}$ are unramified, and we glue together the two preimages (there are two ways to do this, but the choice won't matter). Then $E \cup E^{\prime}$ is a semistable (but not stable) curve which is the special fiber of an admissible double cover $C_{R^{\prime}} \rightarrow \tilde{P}_{R^{\prime}}$. Suppose that the node in the special fiber of $\tilde{P}_{R^{\prime}}$ étale locally is $x y=t^{\ell}$. In a small neighborhood of this node, there are no ramification points. Thus, a small neighborhood of each of these two points of intersection in $C_{R^{\prime}}$ is isomorphic to a small neighborhood of the node in $\tilde{P}_{R^{\prime}}$ and hence étale locally look like $x y=t^{\ell}$. Finally, we have to contract $E^{\prime}$ to a single point to get a stable curve. The result is that the two nodes become one which étale locally looks like $x y=t^{2 \ell}$.

Now consider curves of type (2). Use the notation from the previous case. The semistable model $C_{R^{\prime}}$ over Spec $R^{\prime}$ has a hyperelliptic involution whose quotient is the union of two $\mathbb{P}_{R^{\prime}}^{1}$ 's. At the node of $C_{R^{\prime}}$, which locally looks like $R^{\prime} \llbracket x, y \rrbracket /\left\langle x y-t^{m}\right\rangle$ for some $m$, the involution negates $x$ and $y$ since it preserves the two components of $C_{R^{\prime}}$. The ring of invariants is $R^{\prime} \llbracket u, v \rrbracket /\left\langle u v-t^{2 m}\right\rangle$ where $u=x^{2}, v=y^{2}$. This is the local picture for the nodal genus 0 curve.

The result above can also be deduced from Caporaso's general theory in [14, §2]. For a combinatorial illustration of type (6) see Chan's Figure 1 in [16]. The two leftmost and two rightmost edges in her upstairs graph have been contracted away. What is left is a "barbell" graph with five horizontal edges of lengths $a, a, b, c, c$, mapping harmonically to a downstairs graph of edge lengths $a, 2 b, c$. Here we see both of the stretching factors represented in different parts of this harmonic morphism: a 2-edge cycle of total length $a+a$ maps to an edge of length $a$, and a single edge of length $b$ maps to an edge of length $2 b$ downstairs.

Now we consider (b). We need to argue that the internal edge lengths can be computed 
from the 15 quantities $\operatorname{val}\left(m_{i}\right)$, in a manner that is consistent with the description above. For genus 1 curves this is precisely the consistency between Examples 5.1 and 5.2. We explain this for the case of the snowflake tree (7). Without loss of generality, we assume that $\{1,2\}$, $\{3,4\}$ and $\{5,6\}$ are the neighbors on the tree. If $\nu_{i j}$ is half the distance between leaves $i$ and $j$, computed from the six points as in (5.4), then, for instance, $\operatorname{val}\left(m_{13}\right)=-\nu_{16}-\nu_{24}-\nu_{35}$. A direct calculation on the snowflake tree shows that the three internal edge lengths are

$$
\operatorname{val}\left(m_{2}\right)-\operatorname{val}\left(m_{13}\right), \operatorname{val}\left(m_{6}\right)-\operatorname{val}\left(m_{13}\right), \text { and } \operatorname{val}\left(m_{14}\right)-\operatorname{val}\left(m_{13}\right) .
$$

The edge lengths of the tropical curve

are gotten by doubling these numbers.

At present we do not know the level 3 analogues to the stretching factors $1 / 2$ and 2 we saw in the proof above. Such lattice issues will play a role for the natural map from the tropical Burkhardt quartic onto the tropical moduli space $\mathcal{M}_{2}^{\text {tr }}$. We leave that for future research:

Conjecture 5.4. Let $K$ be a complete nonarchimedean field. There is a commutative square

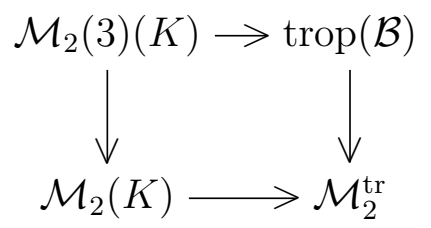

The left map is the forgetful map. The top map is taking valuations of the coordinates $m_{0}, \ldots, m_{39}$. The bottom map sends a curve to its tropical curve. The right map is a morphism of (stacky) fans that takes the third column of Table 3 to the second column.

Here is one concrete way to evaluate the left vertical map $\mathcal{M}_{2}(3) \rightarrow \mathcal{M}_{2}$ over a field $K$. We can represent an element of $\mathcal{M}_{2}(3)$ by a point $\left(r: s_{01}: s_{10}: s_{11}: s_{12}\right) \in \mathbb{P}_{K}^{4}$ that lies in the open Burkhardt quartic $\mathcal{B}^{\circ}$. The corresponding abelian surface $S$ is the singular locus of the Coble cubic in $\mathbb{P}_{K}^{8}$ by Theorem 3.2. If we intersect the abelian surface $S$ with the linear subspace $\mathbb{P}^{4}$ given by (3.6), then the result is the desired genus 2 curve $C \in \mathcal{M}_{2}(K)$. The conjecture asks about the precise relationship between the tropical curve constructed from $C$ and the valuations of our 40 canonical coordinates $m_{0}, m_{1}, \ldots, m_{39}$ on $\mathcal{B}^{\circ}$ inside $\mathbb{P}_{K}^{39}$.

\section{Marked Del Pezzo surfaces}

This section is motivated by our desire to draw all combinatorial types of tropical cubic surfaces together with their 27 lines (trees). These surfaces arise in fibers of the map from a six-dimensional fan to a four-dimensional fan. These tropical moduli spaces were characterized by Hacking, Keel and Tevelev in [33]. We now rederive their fans from first principles.

Consider a reflection arrangement of type $\mathrm{E}_{n}$ for $n=6,7$. The complement of the hyperplanes is the moduli space of $n$ points in $\mathbb{P}^{2}$ in general position (no 2 coincide, no 3 are collinear, no 6 lie on a conic) together with a cuspidal cubic through these points (none of which is the cusp). For $n=6$, there is a 1-dimensional family of such curves (this family is the parabolic curve in [17, Definition 3.2]). For $n=7$ there are 24 choices. We can use maps (1.3) that come from Macdonald representations to forget the data of the cuspidal cubic. 
Consider the case $n=6$. Six points on a cuspidal cubic in $\mathbb{P}^{2}$ are represented by a matrix

$$
D=\left(\begin{array}{cccccc}
1 & 1 & 1 & 1 & 1 & 1 \\
d_{1} & d_{2} & d_{3} & d_{4} & d_{5} & d_{6} \\
d_{1}^{3} & d_{2}^{3} & d_{3}^{3} & d_{4}^{3} & d_{5}^{3} & d_{6}^{3}
\end{array}\right)
$$

The maximal minors of this $3 \times 6$-matrix are denoted

$$
[i j k]=\left(d_{i}-d_{j}\right)\left(d_{i}-d_{k}\right)\left(d_{j}-d_{k}\right)\left(d_{i}+d_{j}+d_{k}\right) \quad \text { for } \quad 1 \leq i<j<k \leq 6 .
$$

We also abbreviate the condition for the six points to lie on a conic:

$$
[\text { conic }]=[134][156][235][246]-[135][146][234][256]=\left(d_{1}+d_{2}+d_{3}+d_{4}+d_{5}+d_{6}\right) \prod_{1 \leq i<j \leq 6}\left(d_{i}-d_{j}\right)
$$

The reflection arrangement of type $\mathrm{E}_{6}$ consists of the $36=\left(\begin{array}{l}6 \\ 3\end{array}\right)+\left(\begin{array}{l}6 \\ 2\end{array}\right)+1$ hyperplanes defined by the linear forms in the products above. We list the flats of this arrangement in Table 4. The bold numbers indicate irreducible flats. Each flat corresponds to a root subsystem, but not conversely. Root subsystems that are not parabolic, such as $\mathrm{A}_{2}^{\times 3}$, do not come from flats.

\begin{tabular}{l|l|l|l|l}
$\#$ & Codim & Size & Root subsystem & Equations of a representative flat \\
\hline $\mathbf{1}$ & 1 & 36 & $\mathrm{~A}_{1}$ & $d_{1}-d_{2}$ \\
\hline $\mathbf{2}$ & 2 & 120 & $\mathrm{~A}_{2}$ & $d_{1}+d_{3}+d_{6}, d_{2}+d_{4}+d_{5}$ \\
3 & 2 & 270 & $\mathrm{~A}_{1} \times \mathrm{A}_{1}$ & $d_{1}+d_{4}+d_{6}, d_{2}+d_{4}+d_{5}$ \\
\hline $\mathbf{4}$ & 3 & 270 & $\mathrm{~A}_{3}$ & $d_{1}+d_{4}+d_{6}, d_{2}+d_{4}+d_{5}, d_{5}-d_{6}$ \\
5 & 3 & 720 & $\mathrm{~A}_{2} \times \mathrm{A}_{1}$ & $d_{1}+d_{5}+d_{6}, d_{2}+d_{4}+d_{5}, d_{4}-d_{5}$ \\
6 & 3 & 540 & $\mathrm{~A}_{1}^{\times 3}$ & $d_{1}+d_{4}+d_{6}, d_{2}+d_{3}+d_{6}, d_{2}+d_{4}+d_{5}$ \\
\hline $\mathbf{7}$ & 4 & 45 & $\mathrm{D}_{4}$ & $d_{5}-d_{6}, d_{3}-d_{4}, d_{2}+d_{4}+d_{6}, d_{1}+d_{4}+d_{6}$ \\
$\mathbf{8}$ & 4 & 216 & $\mathrm{~A}_{4}$ & $d_{5}-d_{6}, d_{3}+d_{4}+d_{6}, d_{2}+d_{4}+d_{6}, d_{1}+d_{4}+d_{6}$ \\
9 & 4 & 540 & $\mathrm{~A}_{3} \times \mathrm{A}_{1}$ & $d_{3}+d_{4}+d_{6}, d_{2}+d_{3}+d_{6}, d_{1}+d_{4}+d_{6}, d_{2}+d_{4}+d_{5}$ \\
10 & 4 & 120 & $\mathrm{~A}_{2} \times \mathrm{A}_{2}$ & $d_{4}-d_{5}, d_{3}+d_{4}+d_{5}, d_{2}+d_{4}+d_{5}, d_{1}+d_{5}+d_{6}$ \\
11 & 4 & 1080 & $\mathrm{~A}_{2} \times \mathrm{A}_{1}^{\times 2}$ & $d_{1}+d_{2}+d_{5}, d_{2}+d_{3}+d_{6}, d_{1}+d_{4}+d_{6}, d_{2}+d_{4}+d_{5}$ \\
\hline $\mathbf{1 2}$ & 5 & 27 & $\mathrm{D}_{5}$ & $d_{5}-d_{6}, d_{1}-d_{4}, d_{1}-d_{3}, d_{1}-d_{2}, d_{1}+d_{5}+d_{6}$ \\
$\mathbf{1 3}$ & 5 & 36 & $\mathrm{~A}_{5}$ & $d_{5}+d_{4}+d_{6}, d_{4}-d_{6}, d_{3}-d_{5}, d_{2}-d_{6}, d_{1}-d_{5}$ \\
14 & 5 & 216 & $\mathrm{~A}_{4} \times \mathrm{A}_{1}$ & $d_{6}, d_{4}, d_{3}-d_{5}, d_{2}+d_{5}, d_{1}$ \\
15 & 5 & 360 & $\mathrm{~A}_{2}^{\times 2} \times \mathrm{A}_{1}$ & $d_{2}+d_{4}+d_{5}, d_{2}-d_{3}, d_{4}-d_{5}, d_{2}+d_{3}+d_{6}, d_{1}+d_{4}+d_{6}$
\end{tabular}

Table 4: The flats of the $\mathrm{E}_{6}$ reflection arrangement.

The Bergman fan of $\mathrm{E}_{6}$ is the fan over the nested set complex [5], a 4-dimensional simplicial complex whose vertices are the $750=36+120+270+45+216+27+36$ irreducible flats.

We define the Yoshida variety $\mathcal{Y}$ to be the closure of the image of the rational map

$$
\mathbb{P}^{5} \stackrel{\text { linear }}{\hookrightarrow} \mathbb{P}^{35} \underset{\substack{\text { monomial } \\-\rightarrow}}{\ln } \mathbb{P}^{39}
$$

where the monomial map is defined by the root subsystems of type $\mathrm{A}_{2}^{\times 3}$. Our name for $\mathcal{Y}$ gives credit to Masaaki Yoshida's explicit computations in [55]. (Warning: there is a closely related variety $\mathcal{Y}$ studied in $[36, \S 3.5]$. This is not the same as our variety.) Explicitly, 
as shown in [17, Proposition 2.4], the map into $\mathbb{P}^{39}$ is defined by 30 bracket monomials like [125][126][134][234][356][456] and 10 bracket monomials like [conic][123][456]. We divide each of these 40 expressions by $\prod_{1<i<j<6}\left(d_{i}-d_{j}\right)$ to get a product of 9 linear forms. Thus the rational map (6.2) is given by 40 polynomials of degree 9 that factor into roots of $\mathrm{E}_{6}$. The tropical Yoshida variety trop $(\mathcal{Y})$ is the image of the Bergman fan of $\mathrm{E}_{6}$ under the linear map $\mathbb{T P}^{35} \rightarrow \mathbb{T P}^{39}$ defined by the corresponding $40 \times 36$-matrix. The Yoshida variety $\mathcal{Y}$ has 40 singular points [53, Theorem 5.7]. Its open part $\mathcal{Y}^{\circ}$ is the moduli space of marked smooth cubic surfaces [17, Theorem 3.1]. The blow-up of these points is Naruki's cross ratio variety $Y_{\mathrm{lc}}^{6}$ (following the notation of [33]) from [42]. The situation is analogous to Theorem 4.7.

As defined, we consider $\operatorname{trop}(\mathcal{Y})$ only as a set, but there is a unique coarsest fan structure on this set. This was shown in [33]. It is the fan over a 3-dimensional simplicial complex that was described by Naruki [42]. We call them the Naruki fan and Naruki complex, respectively. The $76=36+40$ vertices correspond to the two types of boundary divisors on $Y_{\mathrm{lc}}^{6}$ : the 36 divisors coming from the hyperplanes of $\mathrm{E}_{6}$ (type a) and the 40 exceptional divisors of the blow-up (type b). The types of intersections of these divisors is given in [42, p.23] and is listed in Table 5. The divisors of type (a) correspond to root subsystems of type $A_{1}$ and the divisors of type (b) correspond to root subsystems of type $\mathrm{A}_{2}^{\times 3}$. The Naruki complex is the nested set complex on these subsystems. Its face numbers are as follows:

\begin{tabular}{l|l} 
type & number \\
\hline (a) & 36 \\
$(\mathrm{~b})$ & 40 \\
\hline (aa) & 270 \\
$(\mathrm{ab})$ & 360 \\
\hline (aaa) & 540 \\
(aab) & 1080 \\
\hline (aaaa) & 135 \\
(aaab) & 1080
\end{tabular}

Table 5: The Naruki complex has 76 vertices, 630 edges, 1620 triangles and 1215 tetrahedra.

Theorem 6.1. The Yoshida variety $\mathcal{Y}$ is the intersection in $\mathbb{P}^{39}$ of a 9-dimensional linear space and a 15-dimensional toric variety whose dense torus $\mathbb{G}_{m}^{15}$ is the intrinsic torus of $\mathcal{Y}^{\circ}$. The tropical compactification $\overline{\mathcal{Y}}$ of $\mathcal{Y}^{\circ}$ induced by the Naruki fan is the cross ratio variety $Y_{\mathrm{lc}}^{6}$.

The polytope of the toric variety has 2232 facets. Its prime ideal is minimally generated by 8922 binomials, namely 120 of degree 3, 810 of degree 4, 2592 of degree 5, 2160 of degree 6 , and 3240 of degree 8 . These results, which mirror parts (b) and (d) in Proposition 3.1, were found using polymake [28] and gfan [37]. The prime ideal of $\mathcal{Y}$ is minimally generated by 30 of the binomial cubics together with 30 linear forms. A natural choice of such linear forms is described in [55, §3]. It comes from 4-term Plücker relations such as $[123][456]-[124][356]+[125][346]+[126][345]$. There are no linear trinomial relations on $\mathcal{Y}$.

The 750 rays of the $\operatorname{Bergman}$ fan map into $\operatorname{trop}(\mathcal{Y})$ as follows. Write $m$ for the linear map 
$\mathbb{T P}^{35} \rightarrow \mathbb{T P}^{39}$ and $F_{i}$ for the rays representing family $i$ of irreducible flats of Table 4 . Then:

$$
\begin{array}{r}
m\left(F_{1}\right)=m\left(F_{8}\right)=m\left(F_{13}\right) \text { has } 36 \text { elements (a), } \\
m\left(F_{2}\right) \text { has } 40 \text { elements (b), } \\
m\left(F_{4}\right) \text { has } 270 \text { elements. }
\end{array}
$$

All other rays map to 0 in $\mathbb{T P}^{39}$. Each element in $m\left(F_{4}\right)$ is the sum of two vectors from $m\left(F_{1}\right)$ which form a cone. The image of the Bergman fan of $\mathrm{E}_{6}$ in $\mathbb{T P}^{39}$ is a fan with $346=36+40+270$ rays that subdivides the Naruki fan. That fan structure on $\operatorname{trop}(\mathcal{Y})$ defines a modification of the Naruki variety $Y_{\mathrm{lc}}^{6}$.

Here is the finite geometry behind (6.2). Let $V=\mathbb{F}_{2}^{6}$ with coordinates $x_{1}, \ldots, x_{6}$. There are two conjugacy classes of nondegenerate quadratic forms on $V$. Fix the non-split form

$$
q(x)=x_{1} x_{2}+x_{3} x_{4}+x_{5}^{2}+x_{5} x_{6}+x_{6}^{2} .
$$

Then the Weyl group $W\left(\mathrm{E}_{6}\right)$ is the subgroup of $\mathrm{GL}_{6}\left(\mathbb{F}_{2}\right)$ that preserves this form. Using $q(x)$, we define an orthogonal (in characteristic 2 , this also means symplectic) form by

$$
\langle x, y\rangle=q(x+y)-q(x)-q(y) .
$$

There is a natural bijection between the 36 positive roots of $\mathrm{E}_{6}$ and the vectors $x \in V$ with $q(x) \neq 0$. There are 120 planes $W$ such that $q(x) \neq 0$ for all nonzero $x \in W$. These correspond to subsystems of type $\mathrm{A}_{2}$. The set of 120 planes breaks up into 40 triples of pairwise orthogonal planes. These 40 triples correspond to the subsystems of type $\mathrm{A}_{2}^{\times 3}$.

We now come to the case $n=7$. The Göpel variety $\mathcal{G}$ of [44] is the closed image of a map

$$
\mathbb{P}^{6} \stackrel{\text { linear }}{\hookrightarrow} \mathbb{P}^{62} \stackrel{\text { monomial }}{\rightarrow \rightarrow} \mathbb{P}^{134} \text {. }
$$

The linear map is given by the 63 hyperplanes in the reflection arrangement $\mathrm{E}_{7}$, and the monomial map by the 135 root subsystems of type $A_{1}^{\times 7}$. The full list of all flats of the arrangement $\mathrm{E}_{7}$ appears in [44, Table 2]. In [44, Corollary 9.2] we argued that the tropical Göpel variety $\operatorname{trop}(\mathcal{G})$ is the image of the Bergman fan of $\mathrm{E}_{7}$ under the induced linear map $\mathbb{T P}^{62} \rightarrow \mathbb{T P}^{134}$, and we asked how $\operatorname{trop}(\mathcal{G})$ would be related to the fan for $Y_{\mathrm{lc}}^{7}$ in [33, §1.14]. We call that fan the Sekiguchi fan, after [46]. The following theorem answers our question.

Theorem 6.2. The Göpel variety $\mathcal{G}$ is the intersection in $\mathbb{P}^{134}$ of a 14-dimensional linear space and a 35-dimensional toric variety whose dense torus $\mathbb{G}_{m}^{35}$ is the intrinsic torus of $\mathcal{G}^{\circ}$. The tropical compactification $\overline{\mathcal{G}}$ of the open Göpel variety $\mathcal{G}^{\circ}$ induced by the Sekiguchi fan is the Sekiguchi variety $Y_{\mathrm{lc}}^{7}$. Hence, the Sekiguchi fan is the coarsest fan structure on $\operatorname{trop}(\mathcal{G})$.

The result about the linear space and the toric variety is [44, Theorem 6.2]. The determination of the intrinsic tori in Theorems 6.1 and 6.2 is immediate from Lemma 2.6. The last assertion follows from the fact that the open Göpel variety $\mathcal{G}^{\circ}$ is the moduli space of marked smooth del Pezzo surfaces of degree two. For this see [17, Theorem 3.1]. 
The Bergman fan of type $\mathrm{E}_{7}$ has 6091 rays. They are listed in [44, Table 2]. The 6091 rays map into $\operatorname{trop}(\mathcal{G})$ as follows. Write $F_{i}$ for family $i$ in [44, Table 2]. Then:

$$
\begin{array}{r}
m\left(F_{1}\right)=m\left(F_{17}\right)= \\
m\left(F_{25}\right) \text { has } 63 \text { elements, } \\
m\left(F_{15}\right) \text { has } 336 \text { elements, } \\
m\left(F_{4}\right) \text { has } 630 \text { elements, } \\
m\left(F_{24}\right) \text { has } 36 \text { elements, } \\
m\left(F_{8}\right) \text { has } 2016 \text { elements, } \\
m\left(F_{9}\right) \text { has } 315 \text { elements, } \\
m\left(F_{16}\right) \text { has } 1008 \text { elements. }
\end{array}
$$

Finally, $m$ sends $F_{26}$ to 0 (multiple of all 1's vector). The fan on the first 4 types of rays is the Sekiguchi fan as described in $[33, \S 1.14]$. The image of the Bergman fan of $\mathrm{E}_{7}$ is a refinement of the Sekiguchi fan, as follows:

- Every ray in $m\left(F_{8}\right)$ is uniquely the sum of a ray in $m\left(F_{2}\right)$ and a ray in $m\left(F_{24}\right)$. This is in the image of a cone of nested set type $\mathrm{A}_{2} \subset \mathrm{A}_{6}$.

- Every ray in $m\left(F_{9}\right)$ is uniquely the sum of three rays in $m\left(F_{1}\right)$. This is in the image of a cone of nested set type $\mathrm{A}_{1}^{\times 3}$.

- Every ray in $m\left(F_{16}\right)$ can be written uniquely as a positive sum of a ray in $m\left(F_{1}\right)$ and a ray in $m\left(F_{24}\right)$. This is in the image of a cone of nested set type $\mathrm{A}_{1} \subset \mathrm{A}_{6}$.

The Sekiguchi fan on $\operatorname{trop}(\mathcal{G})$ is a fan over a 5 -dimensional simplicial complex with $1065=63+336+630+36$ vertices. It has 9 types of facets, corresponding to the 9 tubings shown in [33, Figure 2, page 200]. The significance of the Naruki fan and the Sekiguchi fan lies in the commutative diagram in [33, Lemma 5.4], which we restate here:

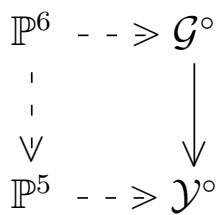

The horizontal maps are those in (6.3) and (6.2). The left vertical map is defined by dropping a coordinate. The tropicalization of the right vertical map $\mathcal{G}^{\circ} \rightarrow \mathcal{Y}^{\circ}$ is a linear projection

$$
\operatorname{trop}(\mathcal{G}) \rightarrow \operatorname{trop}(\mathcal{Y})
$$

from the tropical Göpel variety onto the tropical Yoshida variety.

We wish to explicitly determine this map on each cone of $\operatorname{trop}(\mathcal{G})$. The point is that all tropicalized generic del Pezzo surfaces of degree 3 appear in the fibers of (6.5), by the result about the universal family in [33, Theorem 1.2], and our Theorems 6.1 and 6.2. At infinity, such a del Pezzo surface is glued from 27 trees, which are exactly the tropical image of the 27 lines on a cubic surface over $K$. Each tree has 10 leaves, which come from the intersections of the 27 lines. Thus, each tree represents a point of $\mathcal{M}_{0,10}(K)$. Thus tropicalized del Pezzo surfaces of degree 3 can be represented by a tree arrangement in the sense of [34, §4].

One issue with the map (6.5) is that its zero fiber is 3-dimensional. Namely, it the union of tropicalizations of all constant coefficient cubic surfaces. The zero fiber has 27 rays, one 
for each line on the cubic surface, and 45 triangular cones, one for each triple of pairwise intersecting lines. This is the subtle issue of Eckhart points, addressed by [33, Theorem 1.19]. Cubic surfaces with Eckhart points are special, for they contribute to the points in the interior of the 45 triangular cones. Disallowing these removes the interiors of the triangular cones, and we are left with a balanced two-dimensional fan. This is the fan over a graph with 27 vertices and 135 edges, representing generic constant coefficient cubic surfaces.

In this section, we developed some tools for the classification of tropical cubic surfaces, namely as fibers of (6.5), but we did not actually carry out this classification. That problem will be solved in a forthcoming paper by Qingchun Ren, Kristin Shaw and Bernd Sturmfels.

\section{References}

[1] 4ti2 team: 4ti2-A software package for algebraic, geometric and combinatorial problems on linear spaces, available at www.4ti2.de.

[2] D. Abramovich: Moduli of algebraic and tropical curves, arXiv:1301.0474v1.

[3] D. Abramovich, L. Caporaso and S. Payne: The tropicalization of the moduli space of curves, arXiv: 1212.0373v1.

[4] F. Ardila and C. Klivans: The Bergman complex of a matroid and phylogenetic trees, J. Combin. Theory Ser. B 96 (2006), 38-49, arXiv:math/0311370v2.

[5] F. Ardila, V. Reiner and L. Williams: Bergman complexes, Coxeter arrangements, and graph associahedra, Sém. Lothar. Combin 54A (2005/07), Art. B54A, arXiv:math/0508240v2.

[6] D. Avritzer and H. Lange: The moduli spaces of hyperelliptic curves and binary forms, Math. Z. 242 (2002), no. 4, 615-632, arXiv:math/0109199v1.

[7] M. Baker, S. Payne and J. Rabinoff: Nonarchimedean geometry, tropicalization, and metrics on curves, arXiv: $1104.0320 \mathrm{v} 2$.

[8] C. Birkenhake and H. Lange: Complex Abelian Varieties, Second Edition, Grundlehren 302, Springer Verlag, Berlin, 2004.

[9] M. Bolognesi: On Weddle surfaces and their moduli, Adv. Geom. 7 (2007), no. 1, 113-144, arXiv:math/0601251v2.

[10] A. Borel and L. Ji: Compactifications of Symmetric and Locally Symmetric Spaces, Mathematics: Theory \& Applications. Birkhäuser Boston, Inc., Boston, MA, 2006.

[11] S. Brannetti, M. Melo and F. Viviani: On the tropical Torelli map, Adv. Math., 226 (2011) 2546-2586, arXiv:0907.3324v5.

[12] L. Caporaso: Geometry of tropical moduli spaces and linkage of graphs, J. Combin. Theory Ser. A 119 (2012), no. 3, 579-598, arXiv:1001.2815v5.

[13] L. Caporaso: Algebraic and tropical curves: comparing their moduli spaces, Handbook of Moduli, Volume I. G. Farkas, I. Morrison (Eds.), Advanced Lectures in Mathematics 24 (2012), 119-160, arXiv: $1101.4821 \mathrm{v} 3$.

[14] L. Caporaso: Gonality of algebraic curves and graphs, arXiv:1201.6246v4.

[15] M. Chan: Combinatorics of the tropical Torelli map, Algebra Number Theory 6 (2012) 1133-1169, arXiv: 1012.4539v2.

[16] M. Chan: Tropical hyperelliptic curves J. Algebraic Combinatorics 37 (2013), 331-359, arXiv: $1110.0273 \mathrm{v} 1$.

[17] E. Colombo, B. van Geemen and E. Looijenga: Del Pezzo moduli via root systems, Algebra, Arithmetic, and Geometry: in Honor of Yu. I. Manin. Vol. I, 291-337, Progr. Math. 269, Birkhäuser, Boston, 2009, arXiv: math/0702442v1.

[18] C. De Concini, C. Procesi: Wonderful models of subspace arrangements, Selecta Math. (N.S.) 1 (1995), no. 3, 459-494. 
[19] J. De Jong, N. Shepherd-Barron and A. Van de Ven: On the Burkhardt quartic, Math. Ann. 286 (1990) 309-328.

[20] A. Dickenstein, E. Feichtner and B. Sturmfels: Tropical discriminants, J. Amer. Math. Soc. 20 (2007) 1111-1133, arXiv:math/0510126v3.

[21] I. Dolgachev and D. Lehavi: On isogenous principally polarized abelian surfaces, Curves and abelian varieties, 51-69, Contemp. Math., 465, Amer. Math. Soc., Providence, RI, 2008, arXiv: 0710.1298v3.

[22] I. Dolgachev and D. Ortland: Point sets in projective spaces and theta functions, Astérisque 165 (1988).

[23] N. Elkies: The identification of three moduli spaces, arXiv:math/9905195v1.

[24] E. Feichtner and B. Sturmfels: Matroid polytopes, nested sets and Bergman fans, Port. Math. (N.S.) 62 (2005), no. 4, 437-468, arXiv:math/0411260v1.

[25] T. Fisher: Pfaffian representations of elliptic normal curves, Trans. Amer. Math. Soc. 362 (2010) $2525-2540$.

[26] E. Freitag and R. Salvati Manni: The Burkhardt group and modular forms, Transformation Groups 9 (2004) 25-45.

[27] E. Freitag and R. Salvati Manni: The Burkhardt group and modular forms: 2, Transformation Groups 9 (2004) 237-256.

[28] E. Gawrilow and M. Joswig: polymake: a framework for analyzing convex polytopes, in: Polytopescombinatorics and computation (Oberwolfach, 1997), 43-73, DMV Seminar 29, Birkhäuser, Basel, 2000.

[29] L. Gruson and S. Sam: Alternating trilinear forms on a 9-dimensional space and degenerations of (3,3)-polarized Abelian surfaces, arXiv:1301.5276v1.

[30] L. Gruson, S. Sam and J. Weyman: Moduli of Abelian varieties, Vinberg $\theta$-groups, and free resolutions, Commutative Algebra (edited by Irena Peeva), 419-469, Springer, 2013, arXiv:1203.2575v2.

[31] K. Gunji: Defining equations of the universal abelian surfaces with level three structure, Manuscripta Math. 119 (2006) 61-96.

[32] P. Hacking: The homology of tropical varieties, Collect. Math. 59 (2008), no. 3, 263-273, arXiv:0711.1847v3.

[33] P. Hacking, S. Keel and J. Tevelev: Stable pair, tropical, and log canonical compactifications of moduli spaces of del Pezzo surfaces, Invent. Math. 178 (2009) 173-227, arXiv:math/0702505v2.

[34] S. Hermann, A. Jensen, M. Joswig and B. Sturmfels: How to draw tropical planes, Electron. J. Combin. 16(2) (2009) R6, arXiv:0808.2383v4.

[35] B. Howard, J. Millson, A. Snowden and R. Vakil: The ideal of relations for the ring of invariants of $n$ points on the line, J. Eur. Math. Soc. 14 (2012) 1-60, arXiv:0909.3230v1.

[36] B. Hunt: The Geometry of some Special Arithmetic Quotients, Lecture Notes in Mathematics 1637, Springer-Verlag, Berlin, 1996.

[37] A. Jensen: Gfan, a software system for Gröbner fans and tropical varieties, Available at http://home . imf.au.dk/jensen/software/gfan/gfan.html.

[38] F. Klein: Vorlesungen über das Ikosaeder und die Auflösung der Gleichungen vom fünften Grade, Teubner, Leipzig, 1884.

[39] D. Maclagan and B. Sturmfels: Introduction to Tropical Geometry, book in preparation, http:// homepages.warwick.ac.uk/staff/D.Maclagan/papers/TropicalBook.html

[40] H. Morikawa: On the relation for two-dimensional theta constants of level three, J. Math. Soc. Japan 20 (1968) 248-262.

[41] D. Mumford: The Red Book of Varieties and Schemes, second, expanded edition, Lecture Notes in Mathematics 1358, Springer-Verlag, Berlin, 1999.

[42] I. Naruki: Cross ratio variety as a moduli space of cubic surfaces, with an appendix by Eduard Looijenga, Proc. London Math. Soc. (3) 45 (1982), no. 1, 1-30.

[43] P. Orlik and L. Solomon: Unitary reflection groups and cohomology, Invent. Math. 59 (1980) 77-94.

[44] Q. Ren, S. Sam, G. Schrader and B. Sturmfels: The universal Kummer threefold, Exp. Math. 22 (2013), no. 3, 327-362, arXiv:1208.1229v3. 
[45] F. Rincón: Computing tropical linear spaces, J. Symbolic Comput. 51 (2013) 86-98, arXiv:1109.4130v2.

[46] J. Sekiguchi: Cross ratio varieties for root systems. II. The case of the root system of type $E_{7}$, Kyushu J. Math. 54 (2000), no. 1, 7-37.

[47] G. Shephard and J. Todd: Finite unitary reflection groups, Canad. J. Math. 6 (1954) 274-304.

[48] D. Speyer and B. Sturmfels: The tropical Grassmannian, Adv. Geom. 4 (2004) 389-411, arXiv: math/0304218v3.

[49] W. Stein et al.: Sage Mathematics Software (Version 5.0), The Sage Development Team, 2012, http://www . sagemath.org.

[50] J. Tevelev: Compactifications of subvarieties of tori, Amer. J. Math. 129 (2007) 1087-1104, arXiv: math/0412329v3.

[51] J. Tevelev: Moduli spaces and invariant theory, Lecture Notes, University of Massachusetts, Spring 2011, available at http://www.math.umass.edu/ tevelev/moduli797.pdf.

[52] G. van der Geer: Note on abelian schemes of level three, Math. Ann. 278 (1987), no. 1-4, 401-408.

[53] B. van Geemen: A linear system on Naruki's moduli space of marked cubic surfaces, Internat. J. Math. 13 (2002), no. 2, 183-208, arXiv:math/0101161v1.

[54] F. Viviani: Tropicalizing vs compactifying the Torelli morphism, arXiv:1204.3875v1.

[55] M. Yoshida: $A W\left(E_{6}\right)$-equivariant projective embedding of the moduli space of cubic surfaces, arXiv: math/0002102v1.

Authors' address: Department of Mathematics, University of California, Berkeley, CA 94720, USA. \{qingchun, svs, bernd\}@math. berkeley.edu 